\title{
Synthesis and Cytotoxicity Evaluation of Some Novel Tetrahydronaphthalene-Pyrazole Derivatives
}

\author{
Somaia S. Abd El-Karim", M. Elsadek*, M. Baraka*, M. I. \\ El-Zahar and M. S. Abd Rabou" \\ Therapeutical Chemistry Department, National Research \\ Center, Dokki, Cairo, 12622 and "Medicinal Chemistry \\ Department, Faculty of Pharmacy, Zagazig Univerisity, Egypt.
}

\begin{abstract}
1 -(1,2,3,4-Tetrahydronaphthalen-6-yl)-3- (1,3-diphenyl- $1 H$-pyrazol4-yl)prop-2-en-1-one (3) was synthesized and then treated with various hydrazine hydrate derivatives to afford the corresponding dihydropyrazoles 4-9, respectively. Condensation of pyrazole carbothioamide 9 with $\alpha$-haloketones yielded the corresponding thiazole derivatives 10a,b. Also, the reaction of the chalcone 3 with various sulphonyl hydrazide derivatives produced the corresponding sulphonyl pyrazole derivatives 11a,b, while its reaction with hydroxylamine hydrochloride yielded the isoxazole derivative 12 . Treatment of the chalcone 3 with thiourea or guanidine sulfate gave the pyrimidine derivatives 13 and 14 respectively. Furthermore, the pyrane derivatives 15-17 were obtained by cyclization of the chalcone 3 with malononitrile, ethyl cyanoacetate and/or ethyl acetoacetate respectively. In addition, 2oxopyridine 18 was allowed to react with different alkyl halides to yield the corresponding 2-substituted pyridine derivatives 19-21. Schiff base 23 was obtained by the reaction of carbohydrzide derivative 22 with anisaldehyde. Some of synthesized compounds were evaluated for cytotoxicity activity against HepG2 (liver carcinoma cell line) and MCF-7 (brest carcinoma cell line) using Doxorubicin as a reference drug.
\end{abstract}

Keywords: Tetrahydronaphthalene, Pyrazole, Pyrazoline, Pyrane, Pyridine, Cytotoxic screening, HepG2 and MCF-7.

The burden of cancer is increasing across the world and thus it is the leading cause of deaths in economically developed countries and the second leading cause of deaths in developing countries ${ }^{(1)}$.

Cancer is a disease characterized by a shift in the controlled mechanisms that govern cell proliferation and differentiation. Malignancy is caused by abnormalities in cells, which might be due to inherited genes or caused by outside exposure of the body to chemicals, radiation, or even infectious agents. Several techniques were adopted for the treatment and eradication of cancerous cells. These techniques involved surgery, radiation, immunotherapy, chemotherapy and chemoprevention ${ }^{(2)}$.

\#Corresponding author. Tel.: 002 01002797080; Fax: 0020233370931.

E-mail address: somaia_elkarim@hotmail.com 
Tetrahydronaphthalene derivatives have attracted significant attention in the field of drug discovery because of their wide array of pharmacological activities, including action as non-steroidal glucocorticoid receptor modulators ${ }^{(3)}$, anticancer $^{(4,5)}$, antimicrobial ${ }^{(6)}$, antifungal ${ }^{(7)}$ and anti-HIV effects ${ }^{(8)}$.

Pyrazole derivatives have been the subject of medical research due to their various biological and pharmacological properties such as antitumor ${ }^{(9,10)}$, antimicrobial ${ }^{(11,12)}$, anti-inflammatory ${ }^{(13)}$ and anticonvulsant ${ }^{(14)}$ activities.

Chalcones have attracted much attention due to their diverse biological activities, such as anti-cancer, anti-oxidant, anti-inflammatory, and/or antiinfective activities ${ }^{(15,16)}$. The chalcones which possess $\alpha, \beta$-unsaturated carbonyl group are the convenient intermediates for the synthesis five, six and seven members ${ }^{(17)}$ heterocycles, often have exhibited diverse biological activity.

Molecular hybridization is one of the many strategies, which have been successfully applied for the design and development of new and efficient chemotherapeutic agents ${ }^{(18)}$. Molecular hybridization involves the combination of two or more chemical entities by either linking or fusing each other to form new hybrid moieties ${ }^{(19)}$. The selection of the chemical moieties is based upon their known bio-profiles and it is expected that the hybrid molecules might exhibit additive pharmacological activities ${ }^{(20,21)}$.

In the light of the above findings, we report herein the synthesis of some new tetrahydronaphthalene derivatives bearing pyrazoline, pyrimidine, pyrane, pyrdine and pyrazole moieties and some of the newly synthesized derivatives were tested for anticancer agents against human hepatocelluar carcinoma HepG2 and human breast cancer MCF-7.

\section{Results and Discussion}

\section{Chemistry}

The synthetic strategies adopted for the synthesis of the intermediate and target compounds are depicted in Schemes 1-3. The synthesis of the key intermediate compound 1-(1,2,3,4-tetrahoursydronaphthalen-6-yl)-3-(1,3diphenyl-1H-pyrazol-4-yl) prop-2-en-1-one (3) was achieved according to Claisen-Schmidt condensation, by reacting of 6-acetyl-1,2,3,4-tetrahydronaphthalene (1) with 1,3-diphenyl- $1 H$-pyrazole-4-carboxaldehyde (2) in ethanolic sodium hydroxide. IR spectrum of compound 3 showed a band at $1650 \mathrm{~cm}^{-1}$ due to $\alpha, \beta$ unsaturated ketone. Cyclocondensation of the chalcones with hydrazine hydrate derivatives afforded the corresponding pyrazolines. Thus the treatment of the chalcone 3 with hydrazine hydrate in ethanol led to the formation of the pyrazoline derivative 4 , while in acetic acid yielded the corresponding $\mathrm{N}$-acetyl pyrazoline derivative 5. IR spectrum of pyrazoline derivative 4 revealed the appearance of the absorption band at $3310 \mathrm{~cm}^{-1}$ corresponding to $(\mathrm{NH})$ and the

Egypt. J. Chem. 57, No. 2 (2014) 
disappearance of any band corresponding to $\alpha, \beta$ unsaturated ketone. ${ }^{1} \mathrm{H}-\mathrm{NMR}$ spectrum of pyrazoline derivative 4 showed the signals of $\mathrm{Ha}, \mathrm{Hb}, \mathrm{Hx}$ of pyrazoline ring as doublet of doublet in the regions 3.12-3.20, 3.45-3.55 and 5.13-5.23 ppm, respectively and ${ }^{1} \mathrm{H}-\mathrm{NMR}$ spectrum of pyrazoline derivative 5 showed a singlet signal at $\delta 2.30 \mathrm{ppm}$ referring to $\left(\mathrm{COCH}_{3}\right)$ group.

Also, the chalcone 3 was functionalized by a classical condensation reaction with substituted hydrazines in refluxed ethanol to give the corresponding $\mathrm{N}$ methyl/phenyl pyrazoline derivatives 6a,b. ${ }^{1} \mathrm{H}$ NMR spectrum of 6a showed the appearance of a singlet signal at $\delta 2.90 \mathrm{ppm}$ corresponding to the methyl protons. Furthermore, the reaction of the chalcone 3 with ethyl and/or phenylthiosemicarbazide in ethanol afforded the corresponding substituted thioamide pyrazoline derivatives $7 \mathrm{a}$, b. IR spectra of compounds $7 \mathrm{a}, \mathrm{b}$ showed absorption bands at $3189,3205 \mathrm{~cm}^{-1}$ referring to (NH) groups, while ${ }^{1} \mathrm{H}$ NMR spectrum of 7 a showed quartet and triplet signals at $\delta 3.33,0.94 \mathrm{ppm}$ referring to ethyl protons. In addition the reaction of the chalcone 3 with semicarbazide hydrochloride in ethanol in the presence of sodium acetate afforded amidopyrazoline derivative 8 . IR spectrum of compounds 8 showed absorption bands at $3201,3124 \mathrm{~cm}^{-1}$ referring to $\left(\mathrm{NH}_{2}\right)$ group and at $1651 \mathrm{~cm}^{-1}$ referring to $(\mathrm{C}=\mathrm{O})$ group.

Several authors have reported that the synthesis of 1-(thiazol-2-yl)pyrazolines from the corresponding chalcones. In the same sense, the reaction of the chalcone 3 with thiosemicarbazide in refluxing ethanol afforded 4,5-dihydro-3(1,2,3,4-tetrahydronaphthalen-6-yl)-5-(1,3-diphenyl- $1 H$-pyrazol-4-yl)pyrazole-1carbothioamide (9). IR spectrum of compound 9 showed absorption bands at $3265,3177 \mathrm{~cm}^{-1}$ referring to $\left(\mathrm{NH}_{2}\right)$ group. The reaction of thioamido-pyrazoline 9 with phenacyl bromide and/or 4-bromophenacyl bromide in refluxing ethanol gave the corresponding thiazolyl-pyrazolines 10a,b.

On the other hand, sulphonyl pyrazoline derivatives 11a,b were obtained from the refluxing of the chalcone 3 with benzene and/or tolyl sulphonylhydrazide in ethanol. IR spectra of compounds 11a showed absorption bands at $1357,1173 \mathrm{~cm}^{1}$ referring to $\left(\mathrm{SO}_{2}\right)$ group. ${ }^{1} \mathrm{H}$ NMR spectrum of $11 \mathrm{~b}$ showed a singlet signal at $\delta 2.41 \mathrm{ppm}$ referring to the methyl group. Finally, cyclization of the chalcone 3 with hydroxylamine hydrochloride in ethanolic sodium hydroxide produced 4-(2,3-dihydro-3-(1,2,3,4-tetrahydronaphthalen-6yl)isoxazol-5-yl)-1,3-diphenyl- $1 H$-pyrazole (12). Its IR spectrum revealed the disappearance of any absorption band attributed to $\alpha, \beta$ unsaturated ketone and the mass spectrum showed a peak at 419 referring to the molecular ion peak (Scheme 1). 


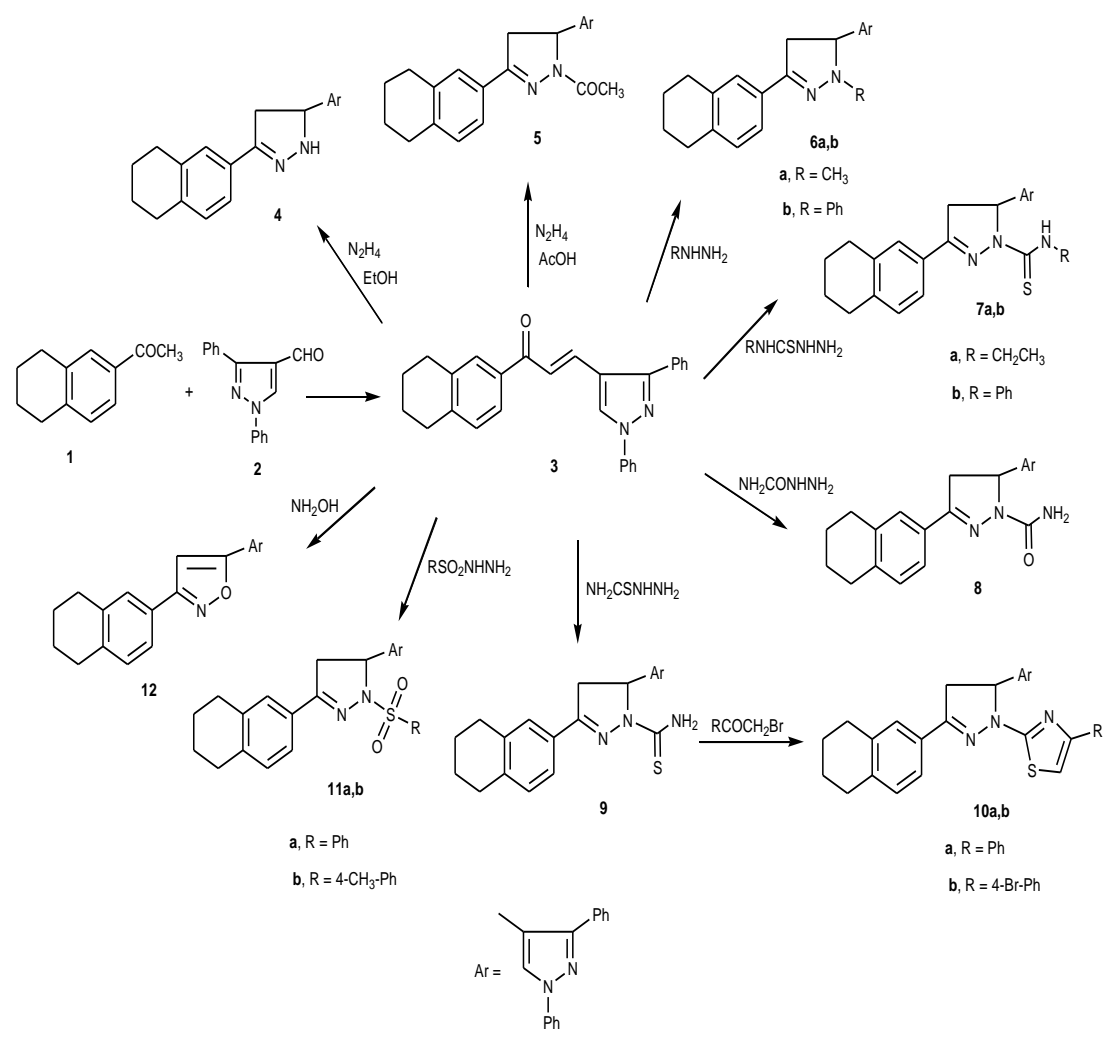

Scheme 1

Moreover, cyclocondensation of the chalcones with different reagents such as thiourea and guanidine afforded the corresponding pyrimidine derivatives. Thus the synthesis of the thiopyrimidine derivative 13 and aminopyrimidine derivative 14 could be successfully achieved by heating a mixture of chalcone 3 with either thiourea or guanidine sulphate in sodium ethoxide, respectively. IR spectrum of compound 13 showed an absorption band at $1159 \mathrm{~cm}^{-1}$ referring to $(\mathrm{C}=\mathrm{S})$ group while IR spectrum of compound 14 showed an absorption band at 3326, 3189 $\mathrm{cm}^{-1}$ referring to $\left(\mathrm{NH}_{2}\right)$ group. Also, the mass spectra of compounds 13 and 14 revealed the molecular ion peaks at 460 and 443 corresponding to the correct molecular formulae of compounds. Also, heating the chalcone 3 with active methylene derivatives namely; malononitrile, ethyl cyanoacetate and/or ethyl acetoacetate in sodium ethoxide solution yielded the targeted pyrane derivatives 15-17, respectively. IR spectrum of pyrane derivative 15 showed absorption bands at $2219 \mathrm{~cm}^{-1}$ referring to $(\mathrm{CN})$ and at $3222,3124 \mathrm{~cm}^{-1}$ referring to $\left(\mathrm{NH}_{2}\right)$ group. Also, IR spectrum of the pyrane derivative 16 showed absorption bands at $2212 \mathrm{~cm}^{-1}$ referring to $(\mathrm{CN})$ and at $3428 \mathrm{~cm}^{-1}$ referring to $(\mathrm{OH})$ group and IR spectrum of the pyrane derivative 17 showed absorption bands at $3423 \mathrm{~cm}^{-1}$

Egypt. J. Chem. 57, No. 2 (2014) 
referring to $(\mathrm{OH})$ and at $1724 \mathrm{~cm}^{-1}$ referring to $\left(\mathrm{COCH}_{3}\right)$ group. The mass spectra of compounds 15-17 revealed the molecular ion peaks at 470, 471 and 488 corresponding to their correct molecular formulae (Scheme 2).

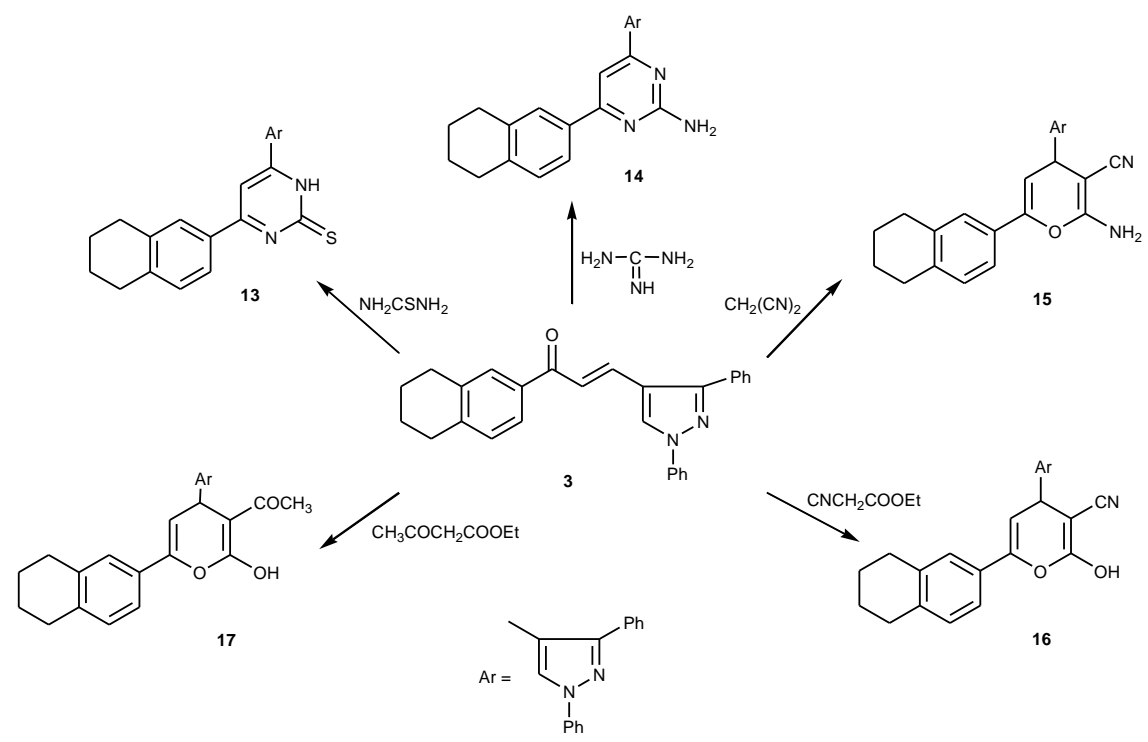

Scheme 2

On the other hand, 1,2-dihydro-6-(1,2,3,4-tetrahydronaphthalen-6-yl)-2-oxo4-(1,3-diphenyl-1H-pyrazol-4-yl)pyridine-3-carbonitrile (18) was synthesized via one-pot multicomponent reaction of 6-acetyl-1,2,3,4-tetrahydronaphthalene (1), 1,3-diphenyl- $1 H$-pyrazole-4-carboxaldehyde (2), ethyl cyanoacetate and excess of ammonium acetate in ethanolic medium. IR spectrum of the pyridone derivative 18 showed absorption bands at 3134,2220 and $1658 \mathrm{~cm}^{-1}$ referring to $\mathrm{NH}_{2}, \mathrm{CN}$ and $\mathrm{CO}$ groups, respectively. Also, the mass spectrum revealed the molecular ion peak at $\mathrm{m} / \mathrm{e} 468$ which is in agreement with its molecular formula.

Different pyridine derivatives could be successfully achieved by the reaction of the pyridone derivative 18 with different reagents. Thus, refluxing of pyridone derivative 18 with methyl iodide and/or chloroacetone in dry dimethylformamide containing anhydrous $\mathrm{K}_{2} \mathrm{CO}_{3}$ yielded the corresponding 2-alkyloxy-pyridine derivatives 19 and 20, respectively. ${ }^{1} \mathrm{H}$ NMR spectrum of compound 19 showed a singlet signal at $\delta \quad 3.71 \mathrm{ppm}$ referring to the methyl group while ${ }^{1} \mathrm{H}$ NMR spectrum of compound 20 showed two singlet signals at $\delta 2.95$ and 2.88 ppm referring to $\left(-\mathrm{CH}_{2}-\right)$ and $\left(\mathrm{CH}_{3}\right)$ groups, respectively. Also, ethyl 2-(3-cyano-6(1,2,3,4-tetrahydronaphthalen-6-yl)-4-(1,3-diphenyl- $1 H$-pyrazol-4-yl)pyridin-2yloxy)acetate (21) was prepared by the treatment of the pyridone derivative 18 with ethyl bromoacetate in dry dimethylformamide containing anhydrous $\mathrm{K}_{2} \mathrm{CO}_{3}$. IR spectrum of the pyridine derivative 21 showed an absorption band at 2219 , 
$1736 \mathrm{~cm}^{-1}$ referring to $(\mathrm{CN})$ and ester groups, while ${ }^{1} \mathrm{H}$ NMR spectrum exhibited the ester group signals at $\delta 1.41$ and $4.38 \mathrm{ppm}$, respectively and the $\left(-\mathrm{CH}_{2}{ }^{-}\right)$ protons appeared as a singlet signal at $\delta 5.13 \mathrm{ppm}$.

Condensation of the pyridine ester derivative 21 with hydrazine hydrate in absolute ethanol afforded the corresponding the pyridine hydrazide derivative 22 . Its IR spectrum showed an absorption band at $3407,3124 \mathrm{~cm}^{-1}$ referring to $\left(\mathrm{NHNH}_{2}\right)$ group. Reaction of the pyridine hydrazide derivative 22 with panisaldehyde in ethanol yielded the corresponding Schiff base 2-(3-cyano-6(1,2,3,4-tetrahydronaphthalen-6-yl)-4-(1,3-diphenyl-1H-pyrazol-4-yl)pyridin-2yloxy)- $N$ '-(4-methoxybenzylidene) acetohydrazide (23) (Scheme 3).

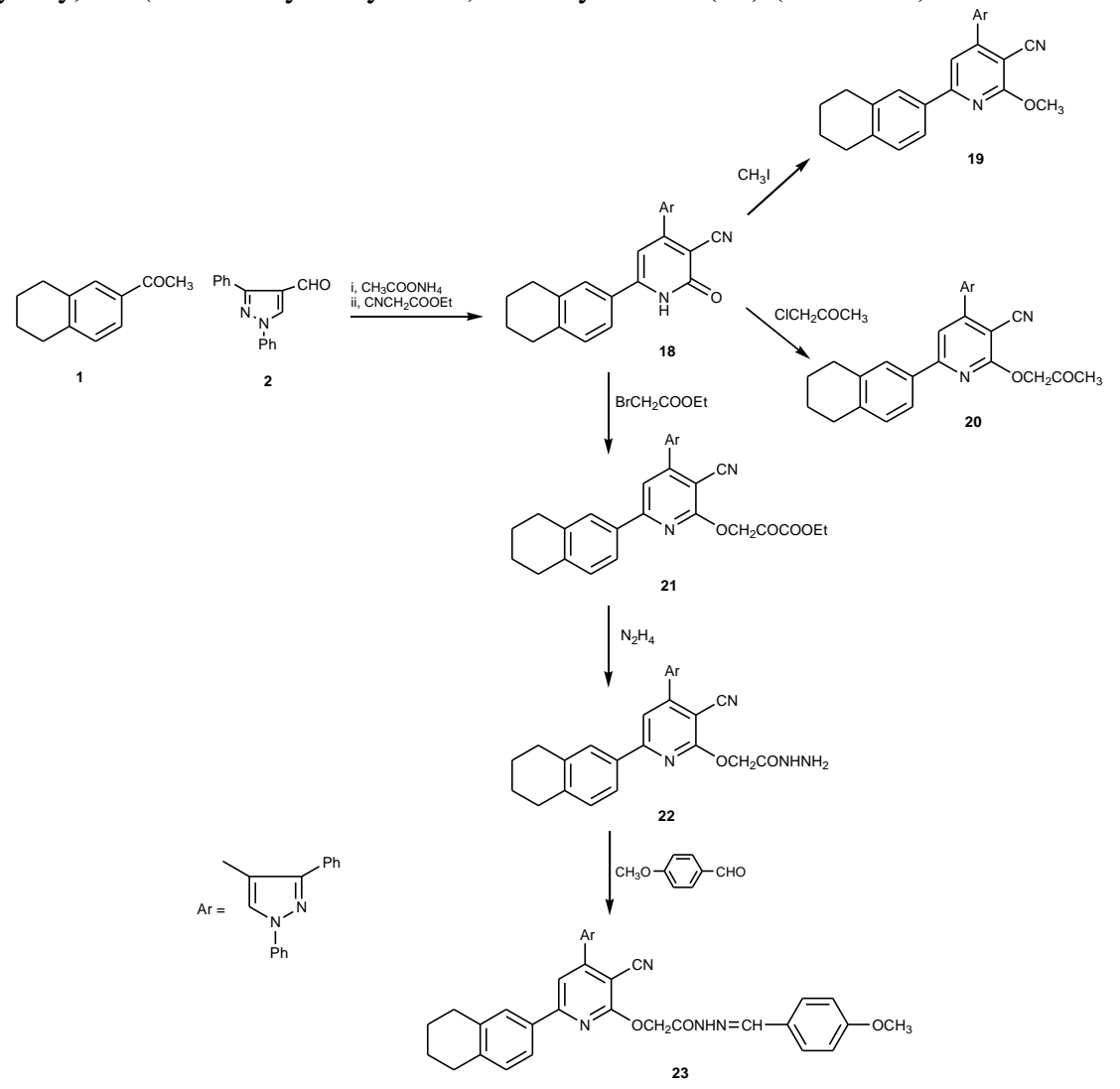

Scheme 3

Cytotoxicity evaluation

The target of this work is to study the cytotoxic activity of the newly synthesized compounds against different carcinoma cell lines and to find the relationship between their chemical structures and their cytotoxic activities. For this reason, fifteen compounds were chosen as examples to examine their in vitro 
potency as cytotoxic agents against human liver carcinoma cell lines (HepG2) and human breast carcinoma cell lines (MCF-7). The cytotoxic activity was expressed as $\mathrm{IC}_{50}$ values measured in $\mu \mathrm{M}$ (the dose that reduces the survival cells to $50 \%$ ), using Doxorubicin as a reference standard.

Regarding to the obtained results in Table $1 \&$ Fig. 1. It has been observed that the combination between tetrahydronaphthalene and pyrazole rings can develop a remarkable cytotoxic activity depending upon the type of the nuclei they attach to. Thus, it can be noticed that the conjugate tetrahydronaphthalene-pyridine-pyrazole as derivative 22 produced the highest cytotoxic activity exceeds that gained by the reference drug Doxorubicin against both types of the examined cell lines $\left(\mathrm{IC}_{50} ; 11\right.$, $6.5,29 \mu \mathrm{M}$, respectively), while the attachment of the hydrazide ethereal linkage of the latter compound to an azomethine side chain as compound 23 greatly reduced the activity against HepG2 cell lines $\left(\mathrm{IC}_{50} ; 63 \mu \mathrm{M}\right)$, but the decrease of the activity was slightly less than that of Doxorubicin $\left(\mathrm{IC}_{50} ; 33 \mu \mathrm{M}\right)$. On the other hand, the conjugation of tetrahydronaphthalene moiety with pyrazolo-2-aminopyrimidine core as compound 14 produced equipotent cytotoxicity to that of Doxorubicin against breast carcinoma cell lines (MCF-7) $\left(\mathrm{IC}_{50} ; 29 \mu \mathrm{M}\right)$, but the activity was 1.5 times less than that of Doxorubicin against liver carcinoma cell lines (HepG2). Remarkable decrease in the cytotoxic activity against both carcinoma cell lines to be twice times less than that of reference drug was obtained upon conversion of the amino group of the latter derivative to the mercapto group as compound $13\left(\mathrm{IC}_{50}\right.$; $60,53 \mu \mathrm{M})$. Unfortunately, the rest of the tested derivatives produced weak potency which requires more structural modifications to improve their cytotoxic activity.

TABLE 1. The effect of the selected compounds on liver carcinoma cell line (HepG2) and Breast carcinoma cell line (MCF-7) compared with Doxorubicin.

\begin{tabular}{|c|c|c|}
\hline \multirow{2}{*}{$\begin{array}{c}\text { Compound } \\
\text { Number }\end{array}$} & \multicolumn{2}{|c|}{ Cell Line } \\
\cline { 2 - 3 } & $\begin{array}{c}\text { HepG2 } \\
\text { IC }_{\mathbf{5 0}}(\boldsymbol{\mu M})\end{array}$ & $\begin{array}{c}\text { MCF-7 } \\
\text { IC }_{\mathbf{5 0}}(\boldsymbol{\mu} \mathbf{M})\end{array}$ \\
\hline Doxorubicin & 29 & 0.24 \\
\hline 3 & 287 & 182 \\
\hline 4 & 218 & 448 \\
\hline 5 & 198 & 346 \\
\hline 8 & 198 & 1000 \\
\hline 9 & 156 & 113 \\
\hline $10 \mathrm{a}$ & 305 & 136 \\
\hline $11 \mathrm{a}$ & 105 & 60 \\
\hline 13 & 60 & 53 \\
\hline 14 & 46 & 29 \\
\hline 16 & 239 & 488 \\
\hline 17 & 167 & 6.5 \\
\hline 22 & 11 & 33 \\
\hline 23 & 63 & 142 \\
\hline
\end{tabular}

$\mathrm{IC}_{50}$ : the dose of the compound which reduces survival to $50 \%$. 


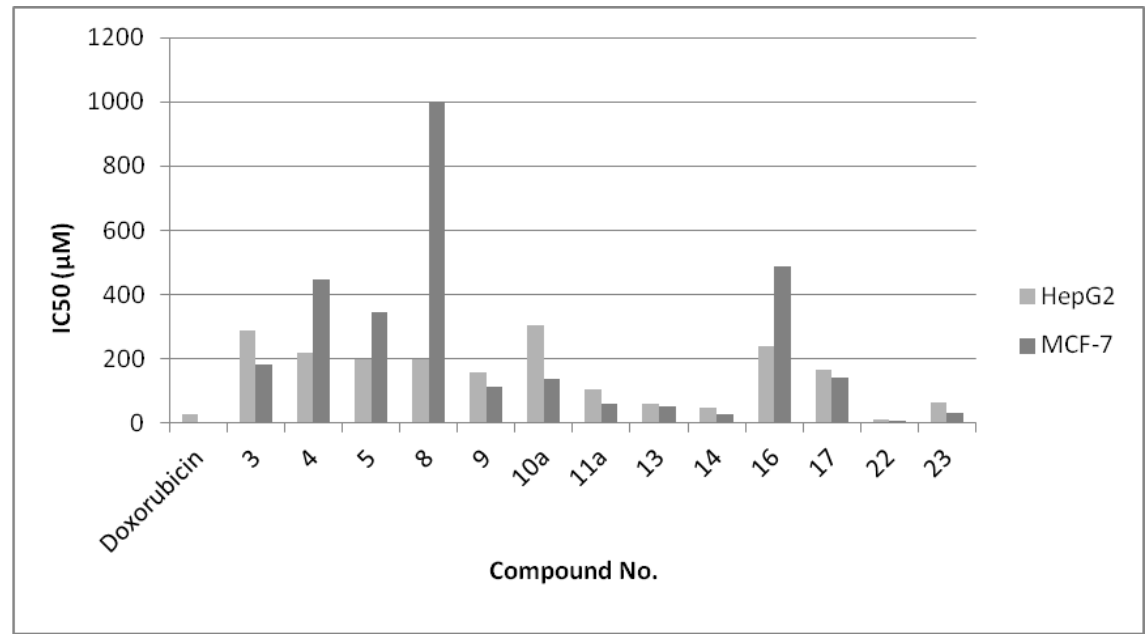

Fig. 1. The effect of the selected compounds on (HepG2) and (MCF-7) compared with Doxorubicin.

\section{Experimental}

All melting points are uncorrected and were taken in open capillary tubes using Electrothermal apparatus 9100. Elemental microanalyses were carried out at Microanalytical Unit, Central Services Laboratory, National Research Centre, Dokki, Cairo, Egypt, using Vario Elementar and were found within $\pm 0.4 \%$ of the theoretical values. Infrared spectra were recorded on a FT/IR-4100 Jasco-Japan, Fourier transform, Infrared spectrometer at $\mathrm{cm}^{-1}$ scale using $\mathrm{KBr}$ disc technique at Central Services Laboratory, National Research Centre, Dokki, Cairo, Egypt. ${ }^{1} \mathrm{H}$ NMR spectra were determined by using a Varian Gemini-200 MHZ NMR spectrometer at Central Services Laboratory, Cairo University, Cairo, Egypt, chemical shifts are expressed in $\delta(\mathrm{ppm})$ downfield from TMS as an internal standard. The mass spectra were measured with a GC MS-Qp1000EX Shimadzu, Cairo University, Cairo, Egypt. Follow up of the reactions and checking the purity of the compounds were made by TLC on silica gel-precoated aluminium sheets (Type 60, F 254, Merck, Darmstadt, Germany) using chloroform/ methanol (5:1, v/v) and the spots were detected by exposure to UV lamp at $\lambda_{254}$ nanometer for few seconds and by iodine vapor.

The chemical names given for the prepared compounds are according to the IUPAC system. 1,3-diphenyl- $1 H$-pyrazole-4-carboxaldehyde (2) was prepared according the reported method ${ }^{(22)}$. 
1-(1,2,3,4-Tetrahydronaphthalen-6-yl)-3-(1,3-diphenyl-1H-pyrazol-4-yl)prop-2en-1-one (3)

A mixture of 6-acetyl-1,2,3,4-tetrahydronaphthalene (1) $(8.7 \mathrm{ml}, 0.05 \mathrm{~mol})$ and 1, 3-diphenyl- $1 H$-pyrazole-4-carboxaldehyde (2) (12.4 g, $0.05 \mathrm{~mol})$ in alcoholic sodium hydroxide $(10 \%, 200 \mathrm{ml})$ was stirred overnight at room temperature. The formed precipitate was filtered, washed several times with water, dried and recrystallized from ethanol to give the title compound 3.

Yield 90\%, mp. 164-165 C; IR (KBr, cm $\left.{ }^{-1}\right): 3057$ (CH-aromatic), 2928, 2864 $\left(\mathrm{CH}_{2}\right.$-tetrahydronaphthalene $), 1650(\mathrm{C}=\mathrm{O}, \alpha, \beta$ unsaturated ketone $), 1582(\mathrm{C}=\mathrm{N})$; ${ }^{1} \mathrm{H} \mathrm{NMR}\left(\mathrm{CDCl}_{3}\right): \delta 1.83\left(\mathrm{~m}, 4 \mathrm{H}, 2 \mathrm{CH}_{2}\right.$-tetrahydronaphthalene protons $), 2.83$ (m, 4H, 2 $\mathrm{CH}_{2}$-tetrahydronaphthalene protons), 7.15-7.90 (m, 15H, CH=CH, Ar$\mathrm{H}), 8.35$ (s, 1H, pyrazole proton); $\mathrm{MS}, \mathrm{m} / \mathrm{z}(\%): 405\left[\mathrm{M}^{+}+1\right](14), 404\left[\mathrm{M}^{+}\right](15)$, $403\left[\mathrm{M}^{+}-1\right](8), 77\left[\mathrm{C}_{6} \mathrm{H}_{5}\right](68), 57\left[\mathrm{C}_{4} \mathrm{H}_{9}\right]$ (100); Anal. For $\mathrm{C}_{28} \mathrm{H}_{24} \mathrm{~N}_{2} \mathrm{O}$ (404.50): Calcd. C, 83.14; H, 5.98; N, 6.93; Found: C, 83.31; H, 6.13; N, 6.71.

4-(4,5-Dihydro-3-(1,2,3,4-tetrahydronaphthalen-6-yl)-1H-pyrazol-5-yl)-1,3-diphenyl -1H-pyrazole (4)

A mixture of compound $3(0.4 \mathrm{~g}, 0.001 \mathrm{~mol})$ and hydrazine hydrate $98 \%$ $(0.10 \mathrm{ml}, 0.002 \mathrm{~mol})$ in absolute ethanol $(10 \mathrm{ml})$ was refluxed for $4 \mathrm{hr}$. The formed precipitate was filtered, dried and recrystallized from ethanol to give the title compound 4 .

Yield 72\%, mp. 179-180 ${ }^{\circ}$; IR ( $\left.\mathrm{KBr}, \mathrm{cm}^{-1}\right): 3310(\mathrm{NH}), 3057$ (CH-aromatic), 2924, $2860\left(\mathrm{CH}_{2}\right.$-tetrahydronaphthalene), $1596(\mathrm{C}=\mathrm{N}) ;{ }^{1} \mathrm{H}$ NMR $\left(\mathrm{CDCl}_{3}\right): \delta 1.82$ $\left(\mathrm{m}, \quad 4 \mathrm{H}, \quad 2 \mathrm{CH}_{2}\right.$-tetrahydronaphthalene protons $), 2.80\left(\mathrm{~m}, 4 \mathrm{H}, 2 \mathrm{CH}_{2}-\right.$ tetrahydronaphthalene protons), 3.12, 3.45 (2dd, $2 \mathrm{H}, \mathrm{CH}_{2}$-pyrazoline protons), 5.13 (dd, 1H, CH-pyrazoline proton), 7.10-8.05 (m, 13H, Ar-H), $8.38(\mathrm{~s}, 1 \mathrm{H}$, pyrazole proton), $9.51\left(\mathrm{~s}, 1 \mathrm{H}, \mathrm{NH}\right.$, exchangeable by $\left.\mathrm{D}_{2} \mathrm{O}\right) ; \mathrm{MS}, \mathrm{m} / \mathrm{z}(\%): 419$ $\left[\mathrm{M}^{+}+1\right](19), 418\left[\mathrm{M}^{+}\right](71), 417\left[\mathrm{M}^{-1}\right](65), 77\left[\mathrm{C}_{6} \mathrm{H}_{5}\right](100)$; Anal. For $\mathrm{C}_{26} \mathrm{H}_{26} \mathrm{~N}_{4}$ (418.53): Calcd. C, 80.35; H, 6.21; N, 13.39; Found: C, 80.52; H, 6.37; N, 13.17.

1-(4,5-Dihydro-3-(1,2,3,4-tetrahydronaphthalen-6-yl)-5-(1,3-diphenyl-1H-pyrazol4-yl) pyrazol-1-yl) ethanone (5).

A mixture of compound $3(0.4 \mathrm{~g}, 0.001 \mathrm{~mol})$ and hydrazine hydrate $98 \%$ $(0.10 \mathrm{ml}, 0.002 \mathrm{~mol})$ in glacial acetic acid $(10 \mathrm{ml})$ was refluxed for $4 \mathrm{hr}$. The formed precipitate was filtered, dried and recrystallized from acetic acid to give the title compound 5 .

Yield 98\%, mp. $220-221^{\circ} \mathrm{C}$; IR ( $\left.\mathrm{KBr}, \mathrm{cm}^{-1}\right): 3046$ (CH-aromatic), 2933, 2860 $\left(\mathrm{CH}_{2}\right.$-tetrahydronaphthalene), $1657(\mathrm{C}=\mathrm{O}), 1591(\mathrm{C}=\mathrm{N}) ;{ }^{1} \mathrm{H}$ NMR $\left(\mathrm{CDCl}_{3}\right): \delta$ $1.75\left(\mathrm{~m}, 4 \mathrm{H}, 2 \mathrm{CH}_{2}\right.$-tetrahydronaphthalene protons), $2.30\left(\mathrm{~s}, 3 \mathrm{H},-\mathrm{COCH}_{3}\right), 2.73$ (m, 4H, 2 $\mathrm{CH}_{2}$-tetrahydronaphthalene protons), 3.16, 3.87 (2dd, 2H, $\mathrm{CH}_{2}-$ pyrazoline protons), 5.68 (dd, 1H, CH-pyrazoline proton), 7.10-7.89 (m, 13H, Ar-H), 8.30 (s, 1H, pyrazole proton); MS, m/z (\%): $461\left[\mathrm{M}^{+}+1\right](24), 460\left[\mathrm{M}^{+}\right]$ 
(67), $459\left[\mathrm{M}^{+}-1\right]$ (31), $77\left[\mathrm{C}_{6} \mathrm{H}_{5}\right]$ (100); Anal. For $\mathrm{C}_{30} \mathrm{H}_{28} \mathrm{~N}_{4} \mathrm{O}$ (460.57): Calcd. C, 78.23; H, 6.13; N, 12.16; Found: C, 78.54; H, 6.28; N, 12.38 .

4-(4,5-Dihydro-3-(1,2,3,4-tetrahydronaphthalen-6-yl)-1-substituted-1H-pyrazol5-yl)-1,3-diphenyl-1H-pyrazole $(6 a, b)$

A mixture of compound $3(0.4 \mathrm{~g}, 0.001 \mathrm{~mol})$, methyl hydrazine and/or phenyl hydrazine $(0.002 \mathrm{~mol})$ in absolute ethanol $(10 \mathrm{ml})$ was refluxed for $3 \mathrm{hr}$. After cooling the formed precipitate was filtered, dried and recrystallized from ethanol to give the title compounds $6 a, b$.

4-(4,5-Dihydro-3- (1,2,3,4-tetrahydronaphthalen-6-yl) -1-methyl-1H-pyrazol-5-yl)1,3-diphenyl-1H-pyrazole (6a)

Yield 58\%, mp. 120-121 ${ }^{\circ} \mathrm{C}$; IR ( $\left.\mathrm{KBr}, \mathrm{cm}^{-1}\right): 3057$ (CH-aromatic), 2926, 2855 $\left(\mathrm{CH}_{2}\right.$-tetrahydronaphthalene), $1597(\mathrm{C}=\mathrm{N}) ;{ }^{1} \mathrm{H} \mathrm{NMR}\left(\mathrm{CDCl}_{3}\right): \delta 1.82(\mathrm{~m}, 4 \mathrm{H}$, $2 \mathrm{CH}_{2}$-tetrahydronaphthalene protons), $2.78\left(\mathrm{~m}, 4 \mathrm{H}, 2 \mathrm{CH}_{2}\right.$-tetrahydronaphthalene protons), 2.90 (s, $3 \mathrm{H}, \mathrm{CH}_{3}$ ), 3.57, 3.97 (2dd, $2 \mathrm{H}, \mathrm{CH}_{2}$-pyrazoline protons), 6.12 (dd, 1H, CH-pyrazoline proton), 7.06-8.07 (m, 13H, Ar-H), 8.30 (s, 1H, pyrazole proton); MS, m/z (\%): $433\left[\mathrm{M}^{+}+1\right](8), 432\left[\mathrm{M}^{+}\right](10), 431\left[\mathrm{M}^{+}-1\right](33), 430\left[\mathrm{M}^{+}-\right.$ 2] (100); Anal. For $\mathrm{C}_{29} \mathrm{H}_{28} \mathrm{~N}_{4}$ (432.56): Calcd. C, 80.52; H, 6.52; N, 12.95; Found: C, 80.34; H, 6.22; N, 13.13.

4-(4,5-Dihydro-3-(1,2,3,4-tetrahydronaphthalen-6-yl)-1-phenyl-1H-pyrazol-5-yl )1,3-diphenyl-1H-pyrazole (6b)

Yield 72\%, mp. 150-151 ${ }^{\circ} \mathrm{C}$; IR $\left(\mathrm{KBr}, \mathrm{cm}^{-1}\right): 3060$ (CH-aromatic), 2922, 2855 $\left(\mathrm{CH}_{2}\right.$-tetrahydronaphthalene), $1594(\mathrm{C}=\mathrm{N}) ;{ }^{1} \mathrm{H} \mathrm{NMR}\left(\mathrm{DMSO}-\mathrm{d}_{6}\right): \delta 1.74(\mathrm{~m}, 4 \mathrm{H}$, $2 \mathrm{CH}_{2}$-tetrahydronaphthalene protons), $2.72\left(\mathrm{~m}, 4 \mathrm{H}, 2 \mathrm{CH}_{2}\right.$-tetrahydronaphthalene protons), 2.96, 3.40 (2dd, $2 \mathrm{H}, \mathrm{CH}_{2}$-pyrazoline protons), 5.00 (dd, $1 \mathrm{H}, \mathrm{CH}-$ pyrazoline proton), 7.04-7.91 (m, 18H, Ar-H), 8.54 (s, 1H, pyrazole proton); MS, $\mathrm{m} / \mathrm{z}(\%): 417\left[\mathrm{M}_{-} \mathrm{C}_{6} \mathrm{H}_{5}\right]^{+}(29), 416\left[\mathrm{M}-\mathrm{C}_{6} \mathrm{H}_{6}\right]^{+}(100)$; Anal. For $\mathrm{C}_{34} \mathrm{H}_{30} \mathrm{~N}_{4}$ (494.63): Calcd. C, 82.56; H, 6.11; N, 11.33; Found: C, 82.29; H, 6.31; N, 11.02.

$N$-substituted-4,5-dihydro-3-(1,2,3,4- tetrahydronaphthalen -6- yl)-5-(1, 3- diphenyl1H-pyrazol-4-yl)pyrazole-1-carbothioamide $(7 a, b)$

A mixture of compound $3(0.4 \mathrm{~g}, 0.001 \mathrm{~mol})$, ethyl thiosemicarbazide and/or phenyl thiosemicarbazide $(0.001 \mathrm{~mol})$ in absolute ethanol $(15 \mathrm{ml})$ was refluxed for $6 \mathrm{hr}$. After cooling the formed precipitate was filtered, dried and recrystallized from the methanol to give the title compounds $7 \mathrm{a}, \mathrm{b}$

$N$-ethyl-4,5-dihydro-3-(1,2,3,4-tetrahydronaphthalen-6-yl)-5-(1,3-diphenyl-1Hpyrazol-4-yl)pyrazole-1-carbothioamide (7a)

Yield 43\%, mp. 196-197 ${ }^{\circ}$; IR ( $\left.\mathrm{KBr}, \mathrm{cm}^{-1}\right): 3189(\mathrm{NH}), 3056$ (CH-aromatic), 2927, $\left.2867\left(\mathrm{CH}_{2} \text {-tetrahydronaphthalene), } 1598(\mathrm{C}=\mathrm{N}) ;{ }^{1} \mathrm{H} \text { NMR (DMSO-d }\right)_{6}\right): \delta$ 0.94 (t, 3H, - $\left.\mathrm{NHCH}_{2} \mathrm{CH}_{3}\right), 1.76\left(\mathrm{~m}, 4 \mathrm{H}, 2 \mathrm{CH}_{2}\right.$-tetrahydronaphthalene protons), $2.80\left(\mathrm{~m}, 4 \mathrm{H}, 2 \mathrm{CH}_{2}\right.$ - tetrahydronaphthalene protons), 3.40, $3.83(2 \mathrm{~m}, 4 \mathrm{H},-$ $\mathrm{NHCH}_{2} \mathrm{CH}_{3}, \mathrm{CH}_{2}$-pyrazoline protons), 5.42 (dd, $1 \mathrm{H}, \mathrm{CH}$-pyrazoline proton), 7.15-7.97 (m, 13H, Ar-H), $8.65(\mathrm{~s}, 1 \mathrm{H}$, pyrazole proton), $9.42(\mathrm{~s}, 1 \mathrm{H}, \mathrm{NH}$,

Egypt. J. Chem. 57, No. 2 (2014) 
exchangeable with $\left.\mathrm{D}_{2} \mathrm{O}\right) ; \mathrm{MS}, \mathrm{m} / \mathrm{z}(\%): 505\left[\mathrm{M}^{+}\right](3), 504\left[\mathrm{M}^{+}-1\right](3), 159$ $\left[\mathrm{C}_{12} \mathrm{H}_{15}\right]$ (100); Anal. For $\mathrm{C}_{31} \mathrm{H}_{31} \mathrm{~N}_{5} \mathrm{~S}$ (505.68): Calcd. C, 73.63; H, 6.18; N, 13.85; Found: C, 73.41; H, 6.29; N, 13.54.

4,5-Dihydro-3-(1,2,3,4-tetrahydronaphthalen-6-yl)-N-phenyl-5-(1,3-diphenyl-1Hpyrazol-4-yl)pyrazole-1-carbothioamide (7b)

Yield 57\%, mp. $200-201^{\circ} \mathrm{C}$; IR (KBr, cm ${ }^{-1}$ ): 3205 (NH), 3063 (CH-aromatic), 2929, $2864\left(\mathrm{CH}_{2}\right.$-tetrahydronaphthalene), $1596(\mathrm{C}=\mathrm{N}) ;{ }^{1} \mathrm{H}$ NMR (DMSO-d $\left.{ }_{6}\right): \delta$ $1.79\left(\mathrm{~m}, 4 \mathrm{H}, 2 \mathrm{CH}_{2}\right.$-tetrahydronaphthalene protons $), 2.85\left(\mathrm{~m}, 4 \mathrm{H}, 2 \mathrm{CH}_{2}-\right.$ tetrahydronaphthalene protons), 3.12, 3.56 (2dd, $2 \mathrm{H}, \mathrm{CH}_{2}$-pyrazoline protons), 5.16 (dd, 1H, CH-pyrazoline proton), 7.04-7.91 (m, 18H, Ar-H), $8.46(\mathrm{~s}, 1 \mathrm{H}$, pyrazole proton), $9.20\left(\mathrm{~s}, 1 \mathrm{H}, \mathrm{NH}\right.$, exchangeable with $\left.\mathrm{D}_{2} \mathrm{O}\right) ; \mathrm{MS}, \mathrm{m} / \mathrm{z}(\%): 554$ $\left[\mathrm{M}^{+}+1\right](11), 553\left[\mathrm{M}^{+}\right](11), 552\left[\mathrm{M}^{-1}\right](13), 77\left[\mathrm{C}_{6} \mathrm{H}_{5}\right](15), 55\left[\mathrm{C}_{4} \mathrm{H}_{7}\right](100)$; Anal. For $\mathrm{C}_{35} \mathrm{H}_{31} \mathrm{~N}_{5} \mathrm{~S}$ (553.72): Calcd. C, 75.92; H, 5.64; N, 12.65; Found: C, 75.62; H, 5.81; N, 12.39 .

4,5-Dihydro-3-(1,2,3,4-tetrahydronaphthalen-6-yl)-5-(1,3-diphenyl-1H-pyrazol4-yl)pyrazole-1-carboxamide (8)

A mixture of compound $3(0.4 \mathrm{~g}, 0.001 \mathrm{~mol})$, semicarbazide hydrochloride $(0.11 \mathrm{~g}, 0.001 \mathrm{~mol})$ and sodium acetate $(0.082 \mathrm{~g}, 0.001 \mathrm{~mol})$ in absolute ethanol $(15 \mathrm{ml})$ was refluxed for $6 \mathrm{hr}$. Then the reaction mixture was poured onto ice/cold water. The formed precipitate was filtered, dried and recrystallized from ethanol to give the title compound 8 .

Yield $70 \%$, mp. $248-249^{\circ} \mathrm{C}$; IR $\left(\mathrm{KBr}, \mathrm{cm}^{-1}\right): 3201,3124\left(\mathrm{NH}_{2}\right), 3061(\mathrm{CH}-$ aromatic), 2931, $2870\left(\mathrm{CH}_{2}\right.$-tetrahydronaphthalene), $1651(\mathrm{C}=\mathrm{O}), 1597(\mathrm{C}=\mathrm{N})$; ${ }^{1} \mathrm{H}$ NMR (DMSO- $\left.\mathrm{d}_{6}\right): \delta 1.76\left(\mathrm{~m}, 4 \mathrm{H}, 2 \mathrm{CH}_{2}\right.$-tetrahydronaphthalene protons), 2.71 (m, 4H, 2 $\mathrm{CH}_{2}$-tetrahydronaphthalene protons), 3.19, 3.42 (2dd, $2 \mathrm{H}, \mathrm{CH}_{2}-$ pyrazoline protons), 5.10 (dd, $1 \mathrm{H}, \mathrm{CH}$-pyrazoline proton), 7.04-7.91 (m, $13 \mathrm{H}$, Ar-H), 8.46 (s, $1 \mathrm{H}$, pyrazole proton), 9.30 (s, $2 \mathrm{H}, \mathrm{NH}_{2}$, exchangeable with $\mathrm{D}_{2} \mathrm{O}$ ); MS, m/z (\%): $417\left[\mathrm{M}^{+}-\mathrm{CONH}_{2}\right](2), 245\left[\mathrm{C}_{14} \mathrm{H}_{19} \mathrm{~N}_{3} \mathrm{O}\right]$ (100), $77\left[\mathrm{C}_{6} \mathrm{H}_{5}\right]$ (64); Anal. For $\mathrm{C}_{29} \mathrm{H}_{27} \mathrm{~N}_{5} \mathrm{O}$ (461.56): Calcd. C, 75.46; H, 5.90; N, 15.17; Found: C, 75.21; H, 5.76; N, 15.29.

4,5-Dihydro-3-(1,2,3,4-tetrahydronaphthalen-6-yl)-5-(1,3-diphenyl-1H-pyrazol4-yl)pyrazole-1-carbothioamide (9)

A mixture of compound $3(1.6 \mathrm{~g}, 0.004 \mathrm{~mol})$ and thiosemicarbazide $(0.36 \mathrm{~g}$, $0.004 \mathrm{~mol})$ in absolute ethanol $(20 \mathrm{ml})$ was refluxed for $10 \mathrm{hr}$. The formed precipitate after cooling was filtered, dried and recrystallized from ethanol to give the title compound 9 .

Yield 78\%, mp. $181-182^{\circ} \mathrm{C}$; IR $\left(\mathrm{KBr}, \mathrm{cm}^{-1}\right): 3265,3177\left(\mathrm{NH}_{2}\right), 3050(\mathrm{CH}-$ aromatic), 2933, $2870\left(\mathrm{CH}_{2}\right.$-tetrahydronaphthalene), $1610(\mathrm{C}=\mathrm{N}) ;{ }^{1} \mathrm{H}$ NMR (DMSO-d $\left.)_{6}\right): \delta 1.76\left(\mathrm{~m}, 4 \mathrm{H}, 2 \mathrm{CH}_{2}\right.$-tetrahydronaphthalene protons), $2.81(\mathrm{~m}, 4 \mathrm{H}$, $2 \mathrm{CH}_{2}$-tetrahydronaphthalene protons), 3.11, 3.40 (2dd, $2 \mathrm{H}, \mathrm{CH}_{2}$-pyrazoline protons), 4.50 (dd, 1H, CH-pyrazoline proton), 7.22-7.96 (m, 14H, Ar-H, 
pyrazole proton), $9.41\left(\mathrm{~s}, 2 \mathrm{H}, \mathrm{NH}_{2}\right.$, exchangeable with $\left.\mathrm{D}_{2} \mathrm{O}\right) ; \mathrm{MS}, \mathrm{m} / \mathrm{z}(\%): 477$ $\left[\mathrm{M}^{+}\right]$(3), $476\left[\mathrm{M}^{+}-1\right](2), 159\left[\mathrm{C}_{12} \mathrm{H}_{15}\right]$ (100); Anal. For $\mathrm{C}_{29} \mathrm{H}_{27} \mathrm{~N}_{5} \mathrm{~S}$ (477.62): Calcd. C, 72.93; H, 5.70; N, 14.66; Found: C, 72.74; H, 5.23; N, 14.83.

4-(4,5-Dihydro-3-(1,2,3,4-tetrahydronaphthalen-6-yl)-1-(4-substituted thiazol-2yl)-1H-pyrazol-5-yl)-1,3-diphenyl-1H-pyrazole $(10 a, b)$

A mixture of compound $9(0.55 \mathrm{~g}, 0.001 \mathrm{~mol})$, phencyl bromide and/or 4bromophencyl bromide $(0.001 \mathrm{~mol})$ in absolute ethanol $(15 \mathrm{ml})$ was refluxed for $5 \mathrm{hr}$. Then the reaction mixture was cooled, poured onto ice/cold water. The formed precipitate was filtered, washed several times by diluted ammonia solution, filtered, dried and recrystallized from ethanol to give the title compounds 10a,b.

4-(4,5-Dihydro-3-(1,2,3,4-tetrahydronaphthalen-6-yl)-1-(4-phenylthiazol-2-yl)1H-pyrazol-5-yl)-1,3-diphenyl-1H-pyrazole (10a)

Yield 71\%, mp. 206-207 ${ }^{\circ} \mathrm{C}$; IR (KBr, $\left.\mathrm{cm}^{-1}\right): 3047$ (CH-aromatic), 2926, 2858 $\left(\mathrm{CH}_{2}\right.$-tetrahydronaphthalene), $1607(\mathrm{C}=\mathrm{N}) ;{ }^{1} \mathrm{H}$ NMR $\left(\right.$ DMSO- $\left.\mathrm{d}_{6}\right)$ : $\delta 1.78(\mathrm{~m}$, $4 \mathrm{H}, \quad 2 \mathrm{CH}_{2}$-tetrahydronaphthalene protons $), \quad 2.80 \quad\left(\mathrm{~m}, \quad 4 \mathrm{H}, \quad 2 \mathrm{CH}_{2-}\right.$ tetrahydronaphthalene protons), 3.01, 3.45 (2dd, $2 \mathrm{H}, \mathrm{CH}_{2}$-pyrazoline protons), 4.19 (dd, $1 \mathrm{H}, \mathrm{CH}$-pyrazoline proton), 6.74-7.95 $(\mathrm{m}, 20 \mathrm{H}, \mathrm{Ar}-\mathrm{H}$, thiazole proton, pyrazole proton); MS, m/z (\%): $578\left[\mathrm{M}^{+}+1\right](36), 577\left[\mathrm{M}^{+}\right](76), 576\left[\mathrm{M}^{+}-1\right]$ (76), $77\left[\mathrm{C}_{6} \mathrm{H}_{5}\right]$ (100); Anal. For $\mathrm{C}_{37} \mathrm{H}_{31} \mathrm{~N}_{5} \mathrm{~S}$ (577.74): Calcd. C, 76.92; H, 5.41; N, 12.12; Found: C, 76.61; H, 5.23; N, 12.39 .

4-(1-(4-(4-Bromophenyl)thiazol-2-yl)-4,5-dihydro-3-(1,2,3,4-tetrahydronaphthalen-6yl)-1H-pyrazol-5-yl)-1,3-diphenyl-1H-pyrazole (10b)

Yield 78\%, mp. 155-156 ${ }^{\circ} \mathrm{C}$; IR (KBr, cm $\left.{ }^{-1}\right): 3057$ (CH-aromatic), 2925, 2857 $\left(\mathrm{CH}_{2}\right.$-tetrahydronaphthalene), $1596(\mathrm{C}=\mathrm{N}) ;{ }^{1} \mathrm{H}$ NMR $\left(\right.$ DMSO- $\left.\mathrm{d}_{6}\right)$ : $\delta 1.76(\mathrm{~m}$, $4 \mathrm{H}, \quad 2 \mathrm{CH}_{2}$-tetrahydronaphthalene protons $), \quad 2.79\left(\mathrm{~m}, \quad 4 \mathrm{H}, \quad 2 \mathrm{CH}_{2-}\right.$ tetrahydronaphthalene protons), 3.18, 3.52 (2dd, $2 \mathrm{H}, \mathrm{CH}_{2}$-pyrazoline protons), 4.24 (dd, $1 \mathrm{H}, \mathrm{CH}$-pyrazoline proton), 6.71-8.01 (m, 19H, Ar-H, thiazole proton, pyrazole proton); MS, m/z (\%): 654, $656\left[\mathrm{M}^{+}-1\right](63,51), 577,575\left[\mathrm{M}^{+}-\mathrm{C}_{6} \mathrm{H}_{6}\right]$ $(54,38), 65\left[\mathrm{C}_{5} \mathrm{H}_{5}\right](100)$; Anal. For $\mathrm{C}_{37} \mathrm{H}_{30} \mathrm{BrN}_{5} \mathrm{~S}$ (656.64): Calcd. C, 67.68; H, 4.61; N, 10.67; Found: C, 67.45; H, 4.29; N, 10.81.

4-(4,5-Dihydro-3-(1,2,3,4-tetrahydronaphthalen-6-yl)-1-(substituted sulphonyl)1H-pyrazol-5-yl)-1,3-diphenyl-1H-pyrazole $(11 a, b)$

A mixture of compound $3(0.4 \mathrm{~g}, 0.001 \mathrm{~mol})$, benzene sulphonyl hydrazide and/or tolyl sulphonyl hydrazide $(0.001 \mathrm{~mol})$ in absolute ethanol $(20 \mathrm{ml})$ was refluxed for $5 \mathrm{hr}$. After cooling the formed precipitate was filtered, dried and recrystallized from methanol to give the title compound 11a,b. 
4-(4,5-Dihydro-3-(1,2,3,4-tetrahydronaphthalen-6-yl)-1-(benzenesulphonyl)-1Hpyrazol-5-yl)-1,3-diphenyl-1H-pyrazole (11a)

Yield 32\%, mp. 187-188 ${ }^{\circ} \mathrm{C}$; IR ( $\left.\mathrm{KBr}, \mathrm{cm}^{-1}\right): 3059$ (CH-aromatic), 2928, 2858 $\left(\mathrm{CH}_{2}\right.$-tetrahydronaphthalene), $1595(\mathrm{C}=\mathrm{N}), 1357,1173\left(\mathrm{SO}_{2}\right) ;{ }^{1} \mathrm{H}$ NMR (DMSO$\left.\mathrm{d}_{6}\right): \delta 1.79\left(\mathrm{~m}, 4 \mathrm{H}, 2 \mathrm{CH}_{2}\right.$-tetrahydronaphthalene protons), $2.81\left(\mathrm{~m}, 4 \mathrm{H}, 2 \mathrm{CH}_{2^{-}}\right.$ tetrahydronaphthalene protons), 3.13, 3.42 (2dd, $2 \mathrm{H}, \mathrm{CH}_{2}$-pyrazoline protons), $5.16(\mathrm{dd}, 1 \mathrm{H}, \mathrm{CH}-$ pyrazoline proton), 7.07-7.99 (m, 18H, Ar-H), $8.36(\mathrm{~s}, 1 \mathrm{H}$, pyrazole proton); MS, m/z (\%): $559\left[\mathrm{M}^{+}+1\right](27), 558\left[\mathrm{M}^{+}\right](39), 557\left[\mathrm{M}^{+}-1\right]$ (27), $55\left[\mathrm{C}_{4} \mathrm{H}_{7}\right]$ (100); Anal. For $\mathrm{C}_{34} \mathrm{H}_{30} \mathrm{~N}_{4} \mathrm{O}_{2} \mathrm{~S}$ (558.69): Calcd. C, 73.09; $\mathrm{H}$, 5.41; N, 10.03; Found: C, 73.32; H, 5.27; N, 10.42.

4-(4,5-Dihydro-3-(1,2,3,4-tetrahydronaphthalen-6-yl)-1-(tolylsulphonyl)-1H-pyrazol-5yl)-1,3-diphenyl-1H-pyrazole (11b)

Yield 57\%, mp. 190-191 ${ }^{\circ} \mathrm{C}$; IR ( $\left.\mathrm{KBr}, \mathrm{cm}^{-1}\right): 3056$ (CH-aromatic), 2926, 2863 $\left(\mathrm{CH}_{2}\right.$-tetrahydronaphthalene), $1597(\mathrm{C}=\mathrm{N}), 1307,1158\left(\mathrm{SO}_{2}\right) ;{ }^{1} \mathrm{H}$ NMR (DMSO$\left.\mathrm{d}_{6}\right): \delta 1.75\left(\mathrm{~m}, 4 \mathrm{H}, 2 \mathrm{CH}_{2}\right.$-tetrahydronaphthalene protons), 2.37 (s, $\left.3 \mathrm{H}, \mathrm{CH}_{3}\right), 2.77$ $\left(\mathrm{m}, 4 \mathrm{H}, 2 \mathrm{CH}_{2}\right.$-tetrahydronaphthalene protons), $3.03,3.39\left(2 \mathrm{dd}, 2 \mathrm{H}, \mathrm{CH}_{2^{-}}\right.$ pyrazoline protons), $5.12(\mathrm{dd}, 1 \mathrm{H}, \mathrm{CH}$-pyrazoline proton), $7.04-7.98(\mathrm{~m}, 17 \mathrm{H}$, Ar-H), 8.48 (s, 1H, pyrazole proton); MS, m/z (\%): $572\left[\mathrm{M}^{+}\right]$(3), $416\left[\mathrm{M}^{+}-\right.$ $\left.\mathrm{C}_{7} \mathrm{H}_{8} \mathrm{O}_{2} \mathrm{~S}\right]$ (70), 159 [ $\left.\mathrm{C}_{12} \mathrm{H}_{15}\right]$ (100); Anal. For $\mathrm{C}_{35} \mathrm{H}_{32} \mathrm{~N}_{4} \mathrm{O}_{2} \mathrm{~S}$ (572.72): Calcd. C, 73.40; H, 5.63; N, 9.78; Found: C, 73.62; H, 5.81; N, 9.49.

4-(3-(1,2,3,4-Tetrahydronaphthalen-6-yl) isoxazol-5-yl) -1,3-diphenyl-1H-pyrazole (12)

A mixture of compound 3 ( $0.8 \mathrm{~g}, 0.002 \mathrm{~mol})$ and hydroxylamine hydrochloride $(0.14 \mathrm{~g}, 0.002 \mathrm{~mol})$ in alcoholic sodium hydroxide $(5 \%, 20 \mathrm{ml})$ was refluxed for 10 hr. The reaction mixture was cooled, poured onto ice/cold water and acidified by diluted hydrochloric acid. The formed precipitate was filtered, dried and recrystallized from ethanol to give the title compound 12.

Yield $85 \%$, mp. $150-151^{\circ} \mathrm{C}$; IR $\left(\mathrm{KBr}, \mathrm{cm}^{-1}\right): 3053$ (CH-aromatic), 2925, 2858 $\left(\mathrm{CH}_{2}\right.$-tetrahydronaphthalene), $1597(\mathrm{C}=\mathrm{N}) ;{ }^{1} \mathrm{H} \mathrm{NMR}\left(\mathrm{CDCl}_{3}\right): \delta 1.81(\mathrm{~m}, 4 \mathrm{H}$, $2 \mathrm{CH}_{2}$-tetrahydronaphthalene protons), $2.80\left(\mathrm{~m}, 4 \mathrm{H}, 2 \mathrm{CH}_{2}\right.$-tetrahydronaphthalene protons), 7.08-8.06 (m, 14H, isoxazole proton, $\mathrm{Ar}-\mathrm{H}), 8.43(\mathrm{~s}, 1 \mathrm{H}$, pyrazole proton); MS, m/z (\%): $419\left[\mathrm{M}^{+}+2\right](25), 418\left[\mathrm{M}^{+}+1\right](20), 417\left[\mathrm{M}^{+}\right]$(9), 77 $\left[\mathrm{C}_{6} \mathrm{H}_{5}\right]$ (100); Anal. For $\mathrm{C}_{28} \mathrm{H}_{23} \mathrm{~N}_{3} \mathrm{O}$ (417.50): Calcd. C, 80.55; H, 5.55; N, 10.06; Found: C, 80.34; H, 5.26; N, 10.36 .

6-(1,2,3,4-Tetrahydronaphthalen-6-yl) -4-(1,3-diphenyl-1H-pyrazol-4-yl) pyrimidine2(1H)-thione (13)

A mixture of compound $2(0.8 \mathrm{~g}, 0.002 \mathrm{~mol})$ and thiourea $(0.23 \mathrm{~g}, 0.002 \mathrm{~mol})$ in sodium ethoxide solution $(5 \%, 15 \mathrm{ml})$ was refluxed for $24 \mathrm{hr}$. The reaction mixture was cooled, poured onto ice/cold water and acidified by diluted hydrochloric acid. The formed precipitate was filtered, dried and recrystallized from ethanol to give the title compound 13 . 
Yield 83\%, mp. 133-134 ${ }^{\circ} \mathrm{C}$; IR (KBr, cm $\left.{ }^{-1}\right): 3417(\mathrm{NH}), 3057$ (CH-aromatic), 2925, $2856\left(\mathrm{CH}_{2}\right.$-tetrahydronaphthalene), $1602(\mathrm{C}=\mathrm{N}), 1159(\mathrm{C}=\mathrm{S}) ;{ }^{1} \mathrm{H}$ NMR $\left(\mathrm{DMSO}_{6}\right): \delta 1.78\left(\mathrm{~m}, 4 \mathrm{H}, 2 \mathrm{CH}_{2}\right.$-tetrahydronaphthalene protons), $2.81(\mathrm{~m}, 4 \mathrm{H}$, $2 \mathrm{CH}_{2}$-tetrahydronaphthalene protons), $5.03(\mathrm{~d}, 1 \mathrm{H}$, pyrimidine proton), 7.16-7.93 $(\mathrm{m}, 14 \mathrm{H}$, pyrimidine proton, $\mathrm{Ar}-\mathrm{H}), 8.52(\mathrm{~s}, 1 \mathrm{H}$, pyrazole proton), $9.21(\mathrm{~s}, 1 \mathrm{H}$, $\mathrm{NH}$, exchangeable with $\left.\mathrm{D}_{2} \mathrm{O}\right) ; \mathrm{MS}, \mathrm{m} / \mathrm{z}(\%): 461\left[\mathrm{M}^{+}+1\right](50), 460\left[\mathrm{M}^{+}\right](70)$, $459\left[\mathrm{M}^{+}-1\right](51), 82\left[\mathrm{C}_{6} \mathrm{H}_{10}\right]$ (100); Anal. For $\mathrm{C}_{29} \mathrm{H}_{24} \mathrm{~N}_{4} \mathrm{~S}$ (460.59): Calcd. C, 75.62; H, 5.25; N, 12.16; Found: C, 75.73; H, 5.43; N, 12.31 .

\section{4-(1,2,3,4-Tetrahydronaphthalen-6-yl)-6- (1,3-diphenyl-1H-pyrazol-4-yl) pyrimidin-2- amine (14)}

A mixture of compound $3(1.6 \mathrm{~g}, 0.004 \mathrm{~mol})$ and guanidine sulfate $(0.43 \mathrm{~g}$, $0.004 \mathrm{~mol})$ in sodium ethoxide solution $(5 \%, 30 \mathrm{ml})$ was refluxed for $24 \mathrm{hr}$. The reaction mixture was cooled, poured onto ice/cold water and acidified by diluted hydrochloric acid. The formed precipitate was filtered, dried and recrystallized from ethanol to give the title compound 14.

Yield $83 \%$, mp. $240-241^{\circ} \mathrm{C}$; IR $\left(\mathrm{KBr}, \mathrm{cm}^{-1}\right): 3326,3189\left(\mathrm{NH}_{2}\right), 3057(\mathrm{CH}-$ aromatic), 2925, $2862\left(\mathrm{CH}_{2}\right.$-tetrahydronaphthalene), $1574(\mathrm{C}=\mathrm{N}) ;{ }^{1} \mathrm{H}$ NMR (DMSO- $\left.\mathrm{d}_{6}\right): \delta 1.80\left(\mathrm{~m}, 4 \mathrm{H}, 2 \mathrm{CH}_{2}\right.$-tetrahydronaphthalene protons), $2.82(\mathrm{~m}, 4 \mathrm{H}$, $2 \mathrm{CH}_{2}$-tetrahydronaphthalene protons), 6.93-7.97 (m, $14 \mathrm{H}$, pyrimidine proton, $\mathrm{Ar}$ $\mathrm{H}), 8.36\left(\mathrm{~s}, 1 \mathrm{H}\right.$, pyrazole proton), $9.23\left(\mathrm{~s}, 2 \mathrm{H}, \mathrm{NH}_{2}\right.$, exchangeable with $\left.\mathrm{D}_{2} \mathrm{O}\right)$; MS, m/z (\%): $444\left[\mathrm{M}^{+}+1\right](15), 443\left[\mathrm{M}^{+}\right](38), 442\left[\mathrm{M}^{+}-1\right](44), 77\left[\mathrm{C}_{6} \mathrm{H}_{5}\right]$ (100), $57\left[\mathrm{C}_{4} \mathrm{H}_{9}\right]$ (100); Anal. For $\mathrm{C}_{29} \mathrm{H}_{25} \mathrm{~N}_{5}$ (443.54): Calcd. C, 78.53; H, 5.68; N, 15.79; Found: C, 78.29; H, 5.42; N, 15.58 .

2-Amino-6-(1,2,3,4-tetrahydronaphthalen-6-yl)-4-(1,3-diphenyl-1H-pyrazol -4-yl)4H-pyran-3-carbonitrile (15), 6-(1,2,3,4-tetrahydronaphthalen -6-yl) -2-hydroxy-4(1,3-diphenyl -1H-pyrazol-4-yl) -4H-pyran-3-carbonitrile (16) and 1-(6-(1,2,3,4tetrahydronaphthalen-6-yl) -2-hydroxy-4- (1,3-diphenyl-1H-pyrazol-4-yl)-4H-pyran 3-yl) ethanone (17)

A mixture of compound $3(0.8 \mathrm{~g}, 0.002 \mathrm{~mol})$ and active methylene compounds namely; malononitrile, ethyl cyanoacetate and/or ethyl acetoacetate $(0.002 \mathrm{~mol})$ in sodium ethoxide solution $(5 \%, 15 \mathrm{ml})$ was refluxed for $6 \mathrm{hr}$. Then the reaction mixture was cooled, poured onto ice/cold water and acidified by diluted hydrochloric acid. The formed precipitate was filtered, dried and recrystallized from ethanol to give the title compounds 15-17.

2-Amino-6- (1,2,3,4-tetrahydronaphthalen -6-yl)-4- (1,3-diphenyl-1H-pyrazol-4yl)- 4H-pyran-3-carbonitrile (15)

Yield 85\%, mp. 208-209 ${ }^{\circ}$; IR $\left(\mathrm{KBr}, \mathrm{cm}^{-1}\right): 3422,3124\left(\mathrm{NH}_{2}\right), 3059(\mathrm{CH}-$ aromatic), 2924, $2855\left(\mathrm{CH}_{2}\right.$-tetrahydronaphthalene), $2219(\mathrm{C} \equiv \mathrm{N}), 1594(\mathrm{C}=\mathrm{N})$; ${ }^{1} \mathrm{H} \mathrm{NMR}\left(\mathrm{CDCl}_{3}\right): \delta 1.81\left(\mathrm{~m}, 4 \mathrm{H}, 2 \mathrm{CH}_{2}\right.$-tetrahydronaphthalene protons), 2.78 $\left(\mathrm{m}, 4 \mathrm{H}, 2 \mathrm{CH}_{2}\right.$-tetrahydronaphthalene protons), 3.90, $4.92(2 \mathrm{~d}, 2 \mathrm{H}$, pyrane protons), 7.11-7.84 (m, 13H, Ar-H), 8.55 (s, 1H, pyrazole proton); MS, m/z (\%): $471\left[\mathrm{M}^{+}+1\right](2), 470\left[\mathrm{M}^{+}\right](3), 444\left[\mathrm{M}^{+}-\mathrm{CN}\right](7), 442$ [444-2] (100); Anal. For

Egypt. J. Chem. 57, No. 2 (2014) 
$\mathrm{C}_{31} \mathrm{H}_{26} \mathrm{~N}_{4} \mathrm{O}$ (470.56): Calcd. C, 79.12; H, 5.57; N, 11.91; Found: C, 79.28; H, 5.31; N, 11.76 .

6-(1,2,3,4-Tetrahydronaphthalen-6-yl)-2-hydroxy-4-(1,3-diphenyl-1H-pyrazol-4yl)-4H-pyran-3-carbonitrile (16)

Yield $85 \%$, mp. $104-105^{\circ} \mathrm{C}$; IR $\left(\mathrm{KBr}, \mathrm{cm}^{-1}\right)$ : Broad band centered at 3428 $(\mathrm{OH}), 3056$ (CH-aromatic), 2925, $2857 \quad\left(\mathrm{CH}_{2}\right.$-tetrahydronaphthalene), 2212 $(\mathrm{C} \equiv \mathrm{N}), \quad 1596 \quad(\mathrm{C}=\mathrm{N}) ; \quad{ }^{1} \mathrm{H} \quad \mathrm{NMR} \quad\left(\right.$ DMSO- $\left._{6}\right): \quad \delta \quad 1.76 \quad\left(\mathrm{~m}, \quad 4 \mathrm{H}, \quad 2 \mathrm{CH}_{2^{-}}\right.$ tetrahydronaphthalene protons), $2.77\left(\mathrm{~m}, 4 \mathrm{H}, 2 \mathrm{CH}_{2}\right.$-tetrahydronaphthalene protons), 3.80, 5.54 (2d, $2 \mathrm{H}$, pyrane protons) 7.02-7.96 (m, 13H, Ar-H), $8.33(\mathrm{~s}$, $1 \mathrm{H}$, pyrazole proton), 12.10 (br. s, $1 \mathrm{H}, \mathrm{OH}$, exchangeable with $\mathrm{D}_{2} \mathrm{O}$ ); $\mathrm{MS}, \mathrm{m} / \mathrm{z}$ (\%): $470\left[\mathrm{M}^{+}-1\right](51), 469\left[\mathrm{M}^{+}-2\right](70), 77\left[\mathrm{C}_{6} \mathrm{H}_{5}\right]$ (100); Anal. For $\mathrm{C}_{31} \mathrm{H}_{25} \mathrm{~N}_{3} \mathrm{O}_{2}$ (471.55): Calcd. C, 78.96; H, 5.34; N, 8.91; Found: C, 78.68; H, 5.08; N, 8.73.

1-(6-(1,2,3,4-Tetrahydronaphthalen-6-yl)-2-hydroxy-4-(1,3-diphenyl-1H-pyrazol-4yl)-4H-pyran-3-yl)ethanone (17)

Yield 95\%, mp. $143-144^{\circ} \mathrm{C}$; IR $\left(\mathrm{KBr}, \mathrm{cm}^{-1}\right)$ : Broad band centered at 3423 $(\mathrm{OH}), 3058$ (CH-aromatic), 2924, $2856\left(\mathrm{CH}_{2}\right.$-tetrahydronaphthalene), 1728 $(\mathrm{C}=\mathrm{O}), \quad 1600 \quad(\mathrm{C}=\mathrm{N}) ;{ }^{1} \mathrm{H} \quad \mathrm{NMR} \quad\left(\right.$ DMSO- $\left._{6}\right): \delta \quad 1.81 \quad\left(\mathrm{~m}, \quad 4 \mathrm{H}, \quad 2 \mathrm{CH}_{2^{-}}\right.$ tetrahydronaphthalene protons), $2.34\left(\mathrm{~s}, 3 \mathrm{H}, \mathrm{COCH}_{3}\right), 2.79\left(\mathrm{~m}, 4 \mathrm{H}, 2 \mathrm{CH}_{2^{-}}\right.$ tetrahydronaphthalene protons), 3.80, $5.70(2 \mathrm{~d}, 2 \mathrm{H}$, pyrane protons) 7.02-7.96 $(\mathrm{m}, 13 \mathrm{H}, \mathrm{Ar}-\mathrm{H}), 8.33$ (s, 1H, pyrazole proton), 12.10 (br. s, $1 \mathrm{H}, \mathrm{OH}$, exchangeable with $\left.\mathrm{D}_{2} \mathrm{O}\right) ; \mathrm{MS}, \mathrm{m} / \mathrm{z}(\%)$ : $487\left[\mathrm{M}^{+}-1\right](12), 91\left[\mathrm{C}_{6} \mathrm{H}_{5} \mathrm{~N}\right](100)$; Anal. For $\mathrm{C}_{32} \mathrm{H}_{28} \mathrm{~N}_{2} \mathrm{O}_{3}$ (488.58): Calcd. C, 78.67; H, 5.78; N, 5.73; Found: C, 78.49; H, 5.61; N, 5.89.

1,2-Dihydro-6-(1,2,3,4-tetrahydronaphthalen-6-yl)-2-oxo-4-(1,3-diphenyl-1H-pyrazol 4-yl)pyridine-3-carbonitrile (18)

A mixture of compound 1 (3.48 g, $0.02 \mathrm{~mol}), 1,3$-diphenyl- $1 H$-pyrazole-4carboxaldehyde (4.96 g, $0.02 \mathrm{~mol})$, ethyl cyanoacetate $(2.26 \mathrm{ml}, 0.02 \mathrm{~mol})$ and ammonium acetate $(12.32 \mathrm{~g}, 0.16 \mathrm{~mol})$ in absolute ethanol $(50 \mathrm{ml})$ was refluxed for $10 \mathrm{hr}$. The formed precipitate was filtered, washed with ethanol, dried and recrystallized from acetic acid to give the title compound 18.

Yield 71\%, mp. $>300^{\circ} \mathrm{C}$; IR $\left(\mathrm{KBr}, \mathrm{cm}^{-1}\right): 3414(\mathrm{NH}), 3061(\mathrm{CH}-$ aromatic) ), 2929, $2866\left(\mathrm{CH}_{2}\right.$-tetrahydronaphthalene), $2220(\mathrm{C} \equiv \mathrm{N}), 1658(\mathrm{C}=\mathrm{O}), 1593(\mathrm{C}=\mathrm{N})$ ; ${ }^{1} \mathrm{H}$ NMR $\left(\mathrm{CDCl}_{3}\right): \delta 1.81$ (m, $4 \mathrm{H}, 2 \mathrm{CH}_{2}$-tetrahydronaphthalene protons), 2.80 (m, 4H, $2 \mathrm{CH}_{2}$-tetrahydronaphthalene protons), 7.15-7.86 (m, $14 \mathrm{H}$, pyridine proton, $\mathrm{Ar}-\mathrm{H}), 8.32(\mathrm{~s}, 1 \mathrm{H}$, pyrazole proton), $9.15(\mathrm{~s}, 1 \mathrm{H}, \mathrm{NH}$, exchangeable with $\left.\mathrm{D}_{2} \mathrm{O}\right) ; \mathrm{MS}, \mathrm{m} / \mathrm{z}(\%): 469\left[\mathrm{M}^{+}+1\right](17), 468\left[\mathrm{M}^{+}\right](45), 467\left[\mathrm{M}^{+}-1\right](16), 77$ $\left[\mathrm{C}_{6} \mathrm{H}_{5}\right]$ (100); Anal. For $\mathrm{C}_{31} \mathrm{H}_{24} \mathrm{~N}_{4} \mathrm{O}$ (468.55): Calcd. C, 79.46; H, 5.16; N, 11.96; Found: C, 79.28; H, 5.31; N, 11.78 . 
6-(1,2,3,4-Tetrahydronaphthalen-6-yl)-2-substituted-4-(1,3-diphenyl-1H-pyrazol-4yl)pyridine-3-carbonitrile (19-21)

A mixture of compound $18(1.88 \mathrm{~g}, 0.004 \mathrm{~mol})$, different alkyl halide namely; methyl iodide, chloroacetone and/or ethyl bromoacetate $(0.004 \mathrm{~mol})$ and potassium carbonate $(0.56 \mathrm{~g}, 0.004 \mathrm{~mol})$ in DMF $(10 \mathrm{ml})$ was refluxed for 8$10 \mathrm{hr}$. Then, the reaction mixture was cooled and poured onto ice/cold water. The formed precipitate was filtered, dried and recrystallized from methanol to give the title compounds 19-21, respectively.

6-(1,2,3,4-Tetrahydronaphthalen-6-yl)-2-methoxy-4-(1,3-diphenyl-1H-pyrazol-4yl)pyridine-3-carbonitrile (19)

Yield 50\%, mp. 187-188 ${ }^{\circ}$; IR $\left(\mathrm{KBr}, \mathrm{cm}^{-1}\right)$ : 3059 (CH-aromatic), 2926, 2859 $\left(\mathrm{CH}_{2}\right.$-tetrahydronaphthalene), $2216(\mathrm{C} \equiv \mathrm{N}), 1590(\mathrm{C}=\mathrm{N}) ;{ }^{1} \mathrm{H} \mathrm{NMR}\left(\mathrm{CDCl}_{3}\right): \delta$ $2.09\left(\mathrm{~m}, 4 \mathrm{H}, 2 \mathrm{CH}_{2}\right.$-tetrahydronaphthalene protons $), 2.96\left(\mathrm{~m}, 4 \mathrm{H}, 2 \mathrm{CH}_{2}-\right.$ tetrahydronaphthalene protons), $3.71\left(\mathrm{~s}, 3 \mathrm{H},-\mathrm{OCH}_{3}\right), 7.28-8.07(\mathrm{~m}, 14 \mathrm{H}$, pyridine proton, $\mathrm{Ar}-\mathrm{H}), 8.46\left(\mathrm{~s}, 1 \mathrm{H}\right.$, pyrazole proton; MS, m/z (\%): $482\left[\mathrm{M}^{+}\right]$ (68), $481\left[\mathrm{M}^{+}-1\right](69), 451\left[\mathrm{M}^{+}-\mathrm{OCH}_{3}\right]$ (79), $77\left[\mathrm{C}_{6} \mathrm{H}_{5}\right]$ (100); Anal. For $\mathrm{C}_{32} \mathrm{H}_{26} \mathrm{~N}_{2} \mathrm{O}$ (482.58): Calcd. C, 79.64; H, 5.43; N, 11.61; Found: C, 79.30; H, $5.71 ; \mathrm{N}, 11.29$.

2-(2-Oxopropoxy)-6-(1,2,3,4-tetrahydronaphthalen-6-yl)-4-(1,3-diphenyl-1H-pyrazol4-yl)pyridine-3-carbonitrile (20)

Yield $75 \%$, mp. $127-128^{\circ} \mathrm{C}$; IR $\left(\mathrm{KBr}, \mathrm{cm}^{-1}\right): 3054$ (CH-aromatic), 2925, 2863 $\left(\mathrm{CH}_{2}\right.$-tetrahydronaphthalene), $2217(\mathrm{C} \equiv \mathrm{N}), 1710(\mathrm{C}=\mathrm{O}), 1602(\mathrm{C}=\mathrm{N}) ;{ }^{1} \mathrm{H}$ NMR $\left(\mathrm{CDCl}_{3}\right): \delta 1.80\left(\mathrm{~m}, 4 \mathrm{H}, 2 \mathrm{CH}_{2}\right.$-tetrahydronaphthalene protons), $2.77(\mathrm{~m}, 4 \mathrm{H}$, $2 \mathrm{CH}_{2}$-tetrahydronaphthalene protons), $2.88\left(\mathrm{~s}, 3 \mathrm{H},-\mathrm{COCH}_{3}\right), 2.95(\mathrm{~s}, 2 \mathrm{H},-$ $\mathrm{OCH}_{2} \mathrm{CO}$ ), 7.11-7.85 (m, 14H, pyridine proton, Ar-H), 8.22 (s, $1 \mathrm{H}$, pyrazole proton); MS, m/z (\%): $525\left[\mathrm{M}^{+}+1\right](81), 524\left[\mathrm{M}^{+}\right](100)$; Anal. For $\mathrm{C}_{34} \mathrm{H}_{28} \mathrm{~N}_{4} \mathrm{O}_{2}$ (524.61): Calcd. C, 77.84; H, 5.38; N, 10.68; Found: C, 77.61; H, 5.45; N, 10.50.

Ethyl 2-(3-cyano-6-(1,2,3,4-tetrahydronaphthalen-6-yl)-4-(1,3-diphenyl-1H-pyrazol 4-yl)pyridin-2-yloxy)acetate (21)

Yield 74\%, mp. $145-146^{\circ} \mathrm{C}$; IR $\left(\mathrm{KBr}, \mathrm{cm}^{-1}\right): 3055$ (CH-aromatic), 2926, 2859 $\left(\mathrm{CH}_{2}\right.$-tetrahydronaphthalene), $2219(\mathrm{C} \equiv \mathrm{N}), 1736(\mathrm{C}=\mathrm{O}$, ester $), 1595(\mathrm{C}=\mathrm{N}) ;{ }^{1} \mathrm{H}$ NMR $\left(\mathrm{CDCl}_{3}\right): \delta 1.41\left(\mathrm{t}, \quad 3 \mathrm{H},-\mathrm{CH}_{2} \mathrm{CH}_{3}\right), \quad 1.81\left(\mathrm{~m}, \quad 4 \mathrm{H}, \quad 2 \mathrm{CH}_{2}\right.$-tetrahy dronaphthalene protons), $2.70\left(\mathrm{~m}, 4 \mathrm{H}, 2 \mathrm{CH}_{2}\right.$-tetrahydronaphthalene protons), $4.38\left(\mathrm{q}, 2 \mathrm{H},-\mathrm{CH}_{2} \mathrm{CH}_{3}\right), 5.13\left(\mathrm{~s}, 2 \mathrm{H},-\mathrm{OCH}_{2}\right), 7.00-7.87$ (m, $14 \mathrm{H}$, pyridine proton, Ar-H), 8.24 (s, 1H, pyrazole proton); MS, m/z (\%): $555\left[\mathrm{M}^{+}+1\right](50), 554\left[\mathrm{M}^{+}\right]$ (19), $553\left[\mathrm{M}^{+}-1\right](15), 77\left[\mathrm{C}_{6} \mathrm{H}_{5}\right](100), 57\left[\mathrm{C}_{4} \mathrm{H}_{9}\right]$ (100); Anal. For $\mathrm{C}_{35} \mathrm{H}_{30} \mathrm{~N}_{4} \mathrm{O}_{3}$ (554.64): Calcd. C, 75.79; H, 5.45; N, 10.10\%; Found: C, 75.92; H, 5.32; N, 10.26 .

2-(3-Cyano-6-(1,2,3,4-tetrahydronaphthalen-6-yl)-4-(1,3-diphenyl-1H-pyrazol4-yl)pyridin-2-yloxy)acetohydrazide (22)

A mixture of compound $20(2.2 \mathrm{~g}, 0.004 \mathrm{~mol})$ and hydrazine hydrate $(0.4 \mathrm{ml}$, $0.008 \mathrm{~mol})$ in absolute ethanol $(20 \mathrm{ml})$ was refluxed for $5 \mathrm{hr}$. The formed

Egypt. J. Chem. 57, No. 2 (2014) 
precipitate was filtered, dried and recrystallized from ethanol to give the title compound 22.

Yield 90\%, mp. 193-194 ${ }^{\circ} \mathrm{C}$; IR $\left(\mathrm{KBr}, \mathrm{cm}^{-1}\right): 3407,3124\left(\mathrm{NH}_{2}, \mathrm{NH}\right), 3056$ (CH-aromatic), 2927, $2861\left(\mathrm{CH}_{2}\right.$-tetrahydronaphthalene), $2219(\mathrm{C} \equiv \mathrm{N}) ;{ }^{1} \mathrm{H} N \mathrm{NMR}$ $\left(\mathrm{CDCl}_{3}\right): \delta 1.82\left(\mathrm{~m}, 4 \mathrm{H}, 2 \mathrm{CH}_{2}\right.$-tetrahydronaphthalene protons), $2.74(\mathrm{~m}, 4 \mathrm{H}$, $2 \mathrm{CH}_{2}$-tetrahydronaphthalene protons), $5.16\left(\mathrm{~s}, 2 \mathrm{H},-\mathrm{OCH}_{2} \mathrm{CO}\right), 7.13-7.84(\mathrm{~m}$, $14 \mathrm{H}$, pyridine proton, $\mathrm{Ar}-\mathrm{H}), 8.60$ (s, $1 \mathrm{H}$, pyrazole proton); $\mathrm{MS}, \mathrm{m} / \mathrm{z}(\%): 540$ $\left[\mathrm{M}^{+}\right]$(73), $539\left[\mathrm{M}^{+}-1\right](44), 109\left[\mathrm{C}_{6} \mathrm{H}_{9} \mathrm{~N}_{2}\right]$ (100); Anal. For $\mathrm{C}_{33} \mathrm{H}_{28} \mathrm{~N}_{6} \mathrm{O}_{2}$ (540.61): Calcd. C, 73.32; H, 5.22; N, 15.55; Found: C, 73.45; H, 5.39; N, 15.23 .

2-(3-Cyano-6-(1,2,3,4-tetrahydronaphthalen-6-yl)-4-(1,3-diphenyl-1H-pyrazol4-yl)pyridin-2-yloxy)-N'-(4-methoxybenzylidene)acetohydrazide (23)

A mixture of compound $22(0.54 \mathrm{~g}, 0.001 \mathrm{~mol})$ and anisaldehyde $(0.14 \mathrm{ml}$, $0.001 \mathrm{~mol})$ in absolute ethanol $(10 \mathrm{ml})$ was refluxed for $8 \mathrm{hr}$. After cooling, the formed precipitate was filtered, dried and recrystallized from ethanol to give the title compound 23.

Yield 67\%, mp. 277-278 ${ }^{\circ} \mathrm{C}$; IR ( $\left.\mathrm{KBr}, \mathrm{cm}^{-1}\right): 3429(\mathrm{NH}), 3058$ (CH-aromatic), 2929, $2857\left(\mathrm{CH}_{2}\right.$-tetrahydronaphthalene $), 2220(\mathrm{C} \equiv \mathrm{N}), 1600(\mathrm{C}=\mathrm{N}) ;{ }^{1} \mathrm{H}$ NMR $\left(\mathrm{CDCl}_{3}\right): \delta 1.79\left(\mathrm{~m}, 4 \mathrm{H}, 2 \mathrm{CH}_{2}\right.$-tetrahydronaphthalene protons), $2.76(\mathrm{~m}, 4 \mathrm{H}$, $2 \mathrm{CH}_{2}$-tetrahydronaphthalene protons), $3.73\left(\mathrm{~s}, 3 \mathrm{H},-\mathrm{OCH}_{3}\right), 5.03(\mathrm{~s}, 2 \mathrm{H},-$ $\left.\mathrm{OCH}_{2} \mathrm{CO}\right), 7.13-7.84(\mathrm{~m}, 19 \mathrm{H}$, pyridine proton, $-\mathrm{N}=\mathrm{CH}, \mathrm{Ar}-\mathrm{H}), 8.39(\mathrm{~s}, 1 \mathrm{H}$, pyrazole proton); MS, m/z (\%): $659\left[\mathrm{M}^{+}+1\right](28), 658\left[\mathrm{M}^{+}\right](52), 77\left[\mathrm{C}_{6} \mathrm{H}_{5}\right]$ (100); Anal. For $\mathrm{C}_{41} \mathrm{H}_{34} \mathrm{~N}_{6} \mathrm{O}_{3}$ (658.75): Calcd. C, 74.75; H, 5.20; N, 12.76; Found: C, 74.56; H, 5.42; N, 12.49 .

\section{Biological assay}

Cytotoxicity assessment

Chemicals: Sulforhodamine-B (SRB) and dimethyl sulfoxide (DMSO) were purchased from Sigma-Aldrich (St. Louis, MO). RPMI-1640 medium, fetal bovine serum and other cell culture materials were purchased from Lonza (Basel, Switzerland).

Cell culture: MCF-7 human breast cancer cell line and HepG2 human hepatocellular carcinoma cancer cells was purchased from VACCERA (Cairo, Egypt) and grown in RPMI-1640 medium and supplemented with $10 \%$ heat inactivated FBS, 100 units $/ \mathrm{ml}$ of penicillin and $100 \mathrm{mg} / \mathrm{ml}$ of streptomycin and maintained at $37^{\circ}$ in a humidified atmosphere containing $5 \% \mathrm{CO}_{2}$.

SRB cytotoxicity assay: Cytotoxicity was determined using SRB method as previously described by Skehan et al ${ }^{(23)}$. Exponentially growing cells were collected using $0.25 \%$ Trypsin-EDTA and seeded in 96-well plates at 2500 cells/well in RPMI-1640 supplemented medium. The cells were allowed for attachment for $24 \mathrm{hr}$. Then, cells were incubated for $72 \mathrm{hr}$ with six different concentrations $\left(0: 10^{3} \mu \mathrm{M}\right)$ of the tested compounds. After $72 \mathrm{hr}$ exposure, the 
cells were fixed with $10 \%$ trichloroacetic acid for $1 \mathrm{hr}$ at $4{ }^{\circ} \mathrm{C}$. The plates were stained for $10 \mathrm{~min}$ at room temperature with $0.4 \%$ sulforhodamine b (SRB) dissolved in $1 \%$ acetic acid. The plates were air dried for $24 \mathrm{hr}$ and the proteindye complex was solubilized with Tris- $\mathrm{HCl}(10 \mathrm{mM}, \mathrm{pH} 7.4)$ for $5 \mathrm{~min}$ on a shaker at $1500 \mathrm{rpm}$. The optical density (OD) was measured spectrophotometrically at $564 \mathrm{~nm}$ using a microplate reader (ChroMate-4300, FL, USA). Half-maximal inhibitory concentration (IC50), the drug concentration at which 50\% growth inhibition is achieved, was calculated using Sigma Plot software, version 9.0 (Systat Software, San Jose, CA).

Acknowledgements: The authors express deepest thanks to Dr. Mai Fathy Tolba, Department of Pharmacology and Toxicology, Faculty of Pharmacy, Ain Shams University and Department of Biology, School of Science and Engineering, American University in Cairo, for carrying out the anticancer screening.

\section{References}

1. Syam, H., Abdelwahab, S.I., Al-Mamary, M.A. and Mohan S., Synthesis of chalcones with anticancer activities. Molecules, 17, 6179-6195 (2012).

2. Al-Omary, F.A.M., Hassan, G. S., El-Messery, S. M. and El Subbagh H. I., Substituted thiazoles V. Synthesis and antitumor activity of novel thiazolo[2,3b]quinazoline and pyrido[4,3-d]thiazolo[3,2-a]pyrimidine analogues. Eur. J. Med. Chem. 47, 65-72 (2012).

3. Barker, M., Clackers, M., Copley, R., Demaine, D.A., Inglis, G.G.A., Johnston, M.J., Jones, H.T., Haas, M.V. House, D. and Loiseau, R., Dissociated nonsteroidal glucocorticoid receptor modulators; discovery of the agonist trigger in a tetrahydronaphthalene-benzoxazine series. J. Med. Chem. 49, 4216-4231(2006).

4. Al-Abdullah E.S., Synthesis and anticancer activity of some novel tetralin-6-ylpyrazoline, 2-thioxopyrimidine, 2-oxopyridine, 2-thioxo-pyridine and 2-iminopyridine derivatives. Molecules, 16, 3410-3419 (2011).

5. Hamdy, N.A., Gamal-Eldeen, A.M., Hatem, A., Abdel-Aziz, H.A. and Fakhr, I.M.I., Modulation of carcinogen metabolizing enzymes by new fused heterocycles pendant to 5,6,7,8-tetrahydronaphthalene derivatives. Eur. J. Med. Chem. 45, 463-470 (2010).

6. Ates-Alagoz, Z., Yildiz, S. and Buyukbingol, E., Antimicrobial activities of some tetrahydronaphthalene-benzimidazole derivatives. Chemotherapy, 53, 110-113 (2007).

7. Tang, H., Zhou, Y.J., Li, Y.W., Lv, J.G., Zheng, C.H., Chen, J. and Zhu, J., Design, synthesis and antifungal activities in vitro of novel tetralin compounds. Chin. Chem. Lett. 19, 264-268 (2008).

8. Al-Mutairi, M.S., Al-Abdullah, E.S., Haiba, M.E., Khedr, M.A. and Zaghary W.A., Synthesis, molecular docking and preliminary in-vitro cytotoxic evaluation of 
some substituted tetrahydronaphthalene (2',3',4',6'-tetra- $O$-acetyl- $\beta$ - D-gluco/galactopyranosyl) derivatives. Molecules, 17, 4717-4732 (2012).

9. Koca, I., Ozgur, A., Coskun,K.A. and Tutar, Y., Synthesis and anticancer activity of acyl thioureas bearing pyrazole moiety. Bioorg. Med. Chem. 21, 3859-3865 (2013).

10. Dawood, K.M., Eldebss, T.M.A., El-Zahabi, H.S.A., Mahmoud H., Yousef, M.H. and Metz, P., Synthesis of some new pyrazole-based 1,3-thiazoles and 1,3,4thiadiazoles as anticancer agents. Eur. J. Med. Chem. 70, 740-749 (2013).

11. Bildirici, I., Sener, A. and Tozlu, I., Further derivatives of 4-benzoyl-1,5-diphenyl$1 H$-pyrazole-3-carboxylic acid and their antibacterial activities. Med. Chem. Res. 16, 418 (2007).

12. Sangani, C.B., Makawana, J.A., Zhang, X., Shashikant, B., Teraiya, S.B., Lin, L. and Hai-Liang, Zhu, Design, synthesis and molecular modeling of pyrazoleequinolinee pyridine hybrids as a new class of antimicrobial and anticancer agents. Eur. J. Med. Chem. 76, 549-557 (2014).

13. Mert, S., Kasımogulları, R., Iça, T., Çolak, F., Altun, A. and Ok, S., Synthesis, structureeactivity relationships and in vitro antibacterial and antifungal activity evaluations of novel pyrazole carboxylic and dicarboxylic acid derivatives. Eur. J. Med. Chem. 78, 86-96 (2014).

14. Ilies, M.A., Masereel, B., Rolin, S., Scozzafava, A., Campeanu, G., Cimpeanu, V. and Supuran, C.T., Carbonic anhydrase inhibitors: aromatic and heterocyclic sulfonamides incorporating adamantyl moieties with strong anticonvulsant activity. Bioorg. Med. Chem. 12, 2717-2726 (2004).

15. Shenvi, S., Kumar, K., Hatti, K.S., Rijesh, K., Diwakar, L. and Chandrasekara Reddy, G., Synthesis, anticancer and antioxidant activities of 2,4,5-trimethoxy chalcones and analogues from asaronaldehyde: Structure-activity relationship. Eur. J. Med. Chem. 62, 435-442 (2013).

16. Mai, C.W., Yaeghoobi, M., Abd-Rahman, N., Kang, Y.B. and Pichika, M.R., Chalcones with electron-withdrawing and electron-donating substituents: Anticancer activity against TRAIL resistant cancer cells, structure-activity relationship analysis and regulation of apoptotic proteins. Eur. J. Med. Chem. 77, 378-387 (2014).

17. Voskiene, A., Mickevicius, V. and Mikulskiene, G., Synthesis and structural characterization of products condensation 4-carboxy-1-(4-styrylcarbonylphenyl)-2pyrrolidinones with hydrazines. ARKIVOC (xv), 303-314 (2007).

18. Viegas-Junior, C., Danuello, A., da Silva Bolzani, V., Barreiro E.J. and Fraga, C.A.M., Molecular hybridization: a useful tool in the design of new drug prototypes. Curr. Med. Chem. 14, 1829-1852 (2007).

19. Walsh, J.J. and Bell, A., Hybrid drugs for malaria. Curr. Pharm. Des. 15, 2970-2985 (2009). 
20. Anand, N., Singh, P., Sharma, A., Tiwari, S., Singh, V., Singh, D.K., Srivastava, K.K., Singh, B.N. and Tripathi, R.P., Synthesis and evaluation of small libraries of triazolylmethoxy chalcones, flavanones and 2-aminopyrimidines as inhibitors of mycobacterial FAS-II and PknG. Bioorg. Med. Chem. 20, 5150-5163 (2012).

21. Karthikeyan, C., Solomon, V.R., Lee, H. and Trivedi, P., Design, synthesis and biological evaluation of some isatin-linked chalcones as novel anti-breast cancer agents: A molecular hybridization approach. Biomedicine \& Preventive Nutrition, $\mathbf{3}$, 325-330 (2013).

22. Ather, A.Q., Tahir, M.N., Khan, M.A., Mehmood, K. and Chaudhry, F., 1,3Diphenyl-1H-pyrazole-4-carboxaldehyde. Acta Cryst. E66, o3170 (2010).

23. Skehan, P., Storeng, R., Scudiero, D., Monks, A., McMahon, J., Vistica, D., Warren, J.T., Bokesch, H., Kenney, S. and Boyd, M.R., New colorimetric cytotoxicity assay for anticancer-drug screening. J. Natl. Cancer Inst. 82,1107-1112 (1990).

(Receied 17/6/2014; accepted 13/7/2014) 


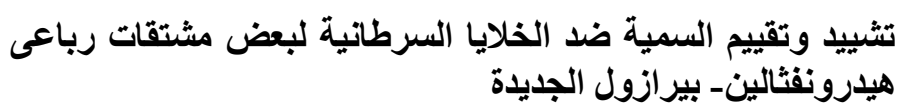

سمية سبد عبد الكريم، محمد الحسينى الصادق*،محمد محمود بركة*،مجدى ابراهيم

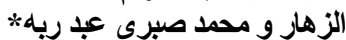

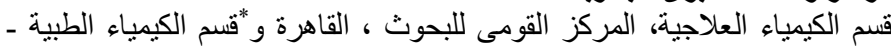
كلية الصيدلة- جامعة الزقازيق- مصر العركي

تناول هذا البحث ثشييد المركب 1- (1,2,3,4-رباعى هيدرونفتالين-6-يل)-3-(1,3-ثنائى فينيل-1 هـ بيرازول-4-يل) بروب-2-ين-1-ين (3) و الذى بدوره نم تفاعله مع مجموعة من مشتقات الهيدرازين هيدرات للحصول على مركبات ثنائى البيرازول المقابلة 4-9. عند تكاثق مركب البيرازول كاربوثيو اميد 9 مع عدد من مشتقات الالفا هالوكيتون ادى الى تكوين مشتقات الثيازول المقابلة 10a,b ـ ايضا عند تفاعل الثالكون 3 مع مجموعة من مشتقات السلفونيل هيدرازيد ادى ذلك للحصول على مركبات السلفونيل

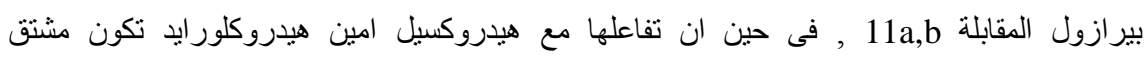
الايزواوكسازول المقابل 12 ـ عند معالجة الثالكون 3 بالثيويوريا وسلفات الجوانيدين ادى الى تكوين

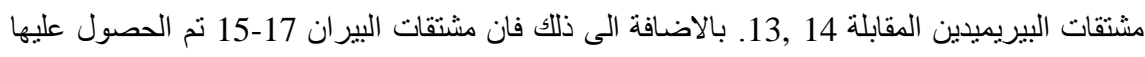
بحولقة الثالكون 3 بالمالونونيتريل, ايثيل سيانواسيتات, ايثيل اسيتو اسيتات. بالاضافة لما سبق تفاعل المركب 2-اوكسوبيريدين 18 مع مجموعة من هاليدات الالكيل المختلفة للحصول على مشتقات البيريدين المقابلة 19-21. عند تفاعل مشتق الكاربو هيدرازيد 22 مع الديهيد الانبسالديهيد تكون مركب الثيف هيف القاعدى المقابل 23 ـ تناولت هذه الدراسة ايضا تقييم سمية بعض المركبات التى تم تشييدها ضد خلايا

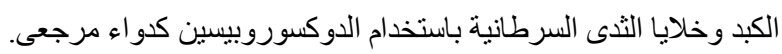

